\title{
Thalamic encoding of lexical status is lateralized during reading aloud
}

Dengyu Wang ${ }^{1,2}$, Witold J. Lipski ${ }^{1 *}$, Alan Bush ${ }^{3,7}$, Anna Chrabaszcz ${ }^{4}$, Christina DastolfoHromack $^{5}$, Michael W. Dickey ${ }^{5}$, Julie A. Fiez ${ }^{4,6}$ \& R. Mark Richardson ${ }^{3,7,8 *}$

1. Department of Neurological Surgery, University of Pittsburgh School of Medicine, Pittsburgh, PA, 15213, USA

2. School of Medicine, Tsinghua University, Beijing, 100084, China

3. Brain Modulation Lab, Massachusetts General Hospital, Boston, MA, 02114, USA

4. Department of Psychology, University of Pittsburgh, Pittsburgh, PA, 15213, USA

5. Department of Communication Science and Disorders, University of Pittsburgh, Pittsburgh, PA, 15213, USA

6. University of Pittsburgh Brain Institute, Pittsburgh, PA, 15213, USA

7. Harvard Medical School, Boston, MA, 02115, USA

${ }^{*}$ For correspondence:

lipskiw@upmc.edu

mark.richardson@mgh.harvard.edu 


\section{Abstract}

To explore whether the thalamus participates in lexical status encoding, local field potentials were recorded in patients undergoing deep brain stimulation lead implantation while they read aloud single-syllable words and nonwords. Bilateral decreases in thalamic beta $(12-30 \mathrm{~Hz})$ activity were preferentially locked to stimulus presentation, and these decreases were greater when nonwords were read. Increased broadband gamma $(70-150 \mathrm{~Hz})$ activity also was locked preferentially to speech onset bilaterally, but greater nonword-related increases in this activity were observed only on the left, demonstrating lateralization of thalamic gamma selectivity for lexical status. In addition, this lexical status effect was strongest in more anterior thalamic locations, regions which are more likely to receive pallidal than cerebellar afferents. These results provide evidence from intracranial thalamic recordings for the lateralization and topography of subcortical lexical status processing. 


\section{Introduction}

2 Reading words aloud depends on our ability to transform information about letter combinations

3 into plans for producing speech sounds. Determining whether a group of letters represents a

4 word is one important component of this process. While word reading can be supported by

5 processes that permit mapping entire word forms to lexical representations, nonword reading

6 depends upon sublexical processes that map spelling and sound (Coltheart et al., 2001).

7 Functional neuroimaging has allowed for increasingly detailed study of the cortical regions that

8 participate in these phonological processes, for instance demonstrating that a region of the

9 inferior frontal gyrus (Brodmann's areas 44 and 45) is significantly more active for nonword

10 reading than for word reading (Fiez et al., 1999; Hagoort et al., 1999; Heim et al., 2005;

11 Herbster et al., 1997). The role of subcortical regions in spoken word production remains

12 elusive, however, due to the low resolution of neuroimaging techniques for measuring

13 subcortical activity. Resolving this knowledge gap is important, given that cortical activity is

14 modulated by thalamic outflow through basal ganglia-thalamo-cortical and cerebello-thalamo-

15 cortical circuits (Behrens et al., 2003; Bosch-Bouju et al., 2013; Hwang et al., 2017; Zhang et

16 al., 2010). Despite the cortico-centric focus of most experimental work and accompanying

17 models, there is increasing recognition of the role of subcortical processes in language

18 production (Llano, 2015).

20 Neurosurgical procedures involving invasive recording and stimulation in epilepsy patients

21 undergoing electrode implantation for seizure mapping traditionally have provided the only direct

22 means to test hypotheses related to cortical function during reading (Juphard et al., 2011).

23 Epilepsy surgery, however, rarely provides access to the thalamus and basal ganglia.

24 Movement disorders surgery, on the other hand, routinely provides direct access to the

25 thalamus and basal ganglia in awake patients. Regionalization of language function within the

26 left thalamus was established in surgery for movement disorders in the 1970s, in seminal

27 studies by Ojemann and colleagues that employed electrical stimulation principles borrowed

28 from traditional cortical language mapping protocols (Johnson \& Ojemann, 2000). More

29 recently, event-related potential recordings in patients undergoing deep brain stimulation (DBS)

30 have suggested that thalamic structures are engaged in the analysis of syntactic, semantic and

31 lexical information during acoustically presented language tasks (Tiedt et al., 2017; Wahl et al.,

32 2008). 
34 We recently developed a protocol to study subcortical activity during single-syllable word/nonword reading, in patients undergoing DBS lead implantation (Chrabaszcz et al., 2019; Lipski et al., 2018). Here, we explore whether the thalamus participates in the encoding of lexical status, by recording local field potentials (LFP) in patients undergoing DBS lead implantation for essential tremor targeting the ventral intermediate nucleus of the thalamus (Vim, which corresponds to the ventral portion of the ventral lateral posterior nucleus (VLp) (Macchi \& Jones, 1997)). Subjects performed a reading aloud task where they were asked to read aloud single-syllable words or nonwords that appeared on a computer screen (Figure 1). We assessed thalamic participation in lexical status encoding by comparing task-related neural responses when participants spoke nonwords vs. words.

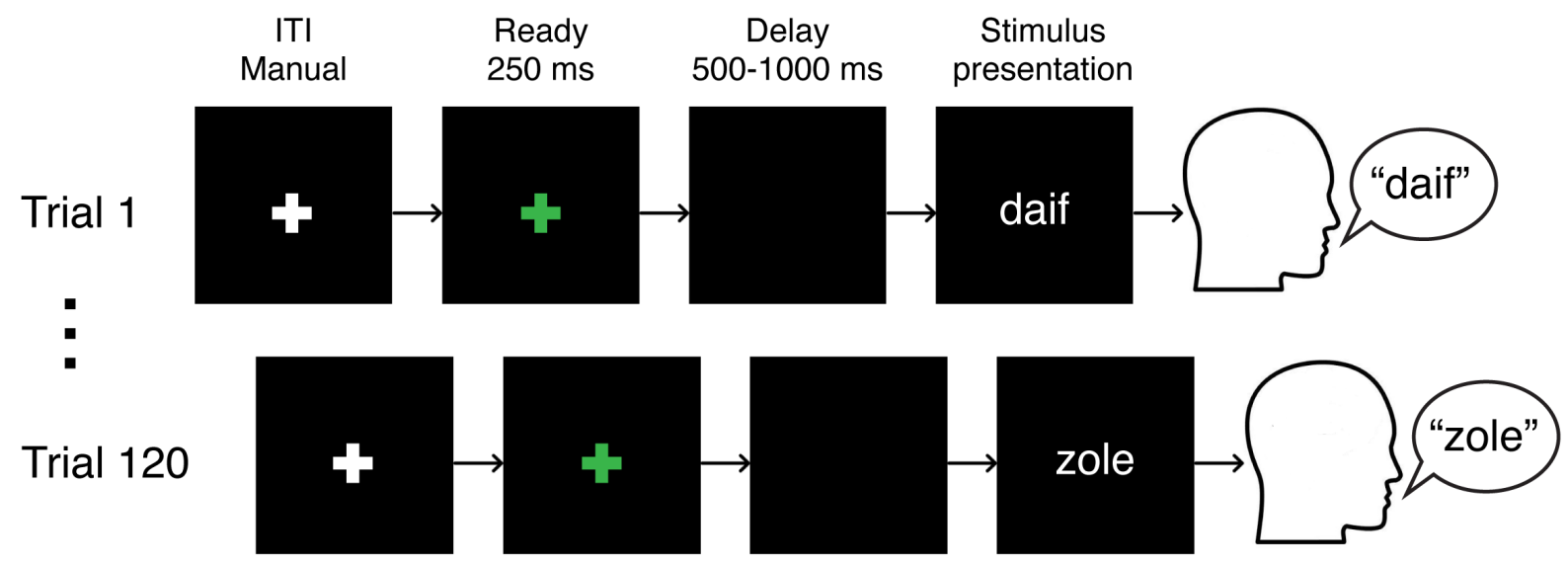

Figure 1: Schematic of experiment. ITI: inter-trial interval.

\section{Results}

49 Eleven subjects read aloud single-syllable words alternating with nonwords, intraoperatively during implantation of DBS leads targeting the Vim nucleus of the thalamus. LFP recordings in four subjects were obtained unilaterally (3 left, 1 right) during microelectrode mapping, where each of these subjects performed up to four sessions of 120 trials. Recordings from seven subjects were obtained from DBS lead contacts, where each of these subjects performed two sessions: in the first session unilateral recordings were made from the left thalamus, and in the second session bilateral recordings were preformed simultaneously. A total of 117 recordings (data recorded in one location in one session) from 89 recording sites pooled across subjects were collected. Recording locations were determined in MNI (Montreal Neurological Institute) space (Figure 2) and comprised locations within or bordering (within $1 \mathrm{~mm}$ ) the ventral anterior nucleus (VA) and the ventral lateral anterior nucleus (VLa) (38/89), or the VLp (51/89). 
a

a

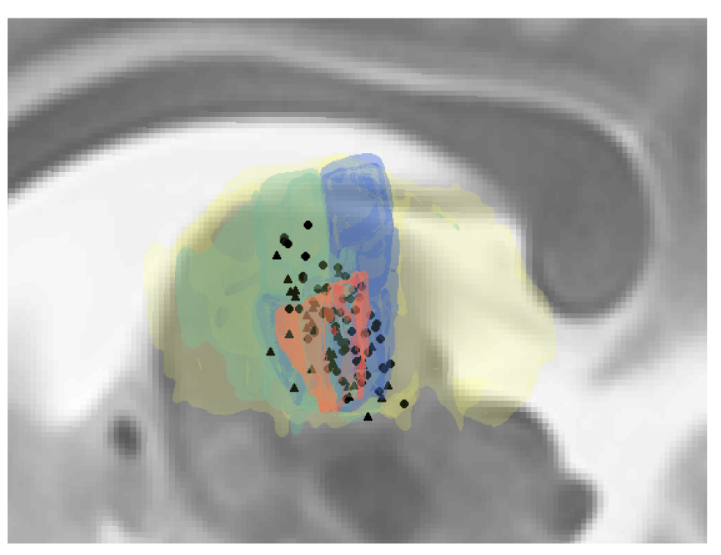

Figure 2: Localization and MNI-transformation of recording sites. a Sagittal view of the recording locations of all the subjects relative to the thalamus (yellow), VA and VLa (green), VLp (blue), and Vim (orange), superimposed on a T2-weighted image. b A plot of all the recording sites in MNI space with their MNI-defined coordinates. Recording contacts of different subjects are color-coded. In both $\mathbf{a}$ and $\mathbf{b}$, right hemisphere recording locations are flipped to the left hemisphere: left side contacts are marked with circles, and right side contacts are marked with triangles.

\section{Behavioral performance}

Across all subjects, the mean speech production latency (interval between stimulus presentation and onset of speech) was $1.07 \pm 0.29 \mathrm{~s}$, and the mean duration of speech was $0.70 \pm 0.09 \mathrm{~s}$.

Nonword production latency $(1.23 \pm 0.37 \mathrm{~s})$ was significantly longer than word production latency $(0.98 \pm 0.24 \mathrm{~s})$ across subjects (two-tailed paired t-test, $\mathrm{t}(10)=4.47, P=0.0012$; Figure $3 \mathrm{a}$ ).

Nonword production duration across subjects $(0.68 \pm 0.07 \mathrm{~s})$ was not significantly different from word production duration $(0.72 \pm 0.13 \mathrm{~s}$; two-tailed paired t-test, $\mathrm{t}(10)=1.99, P=0.074$; Figure $3 \mathrm{~b})$.

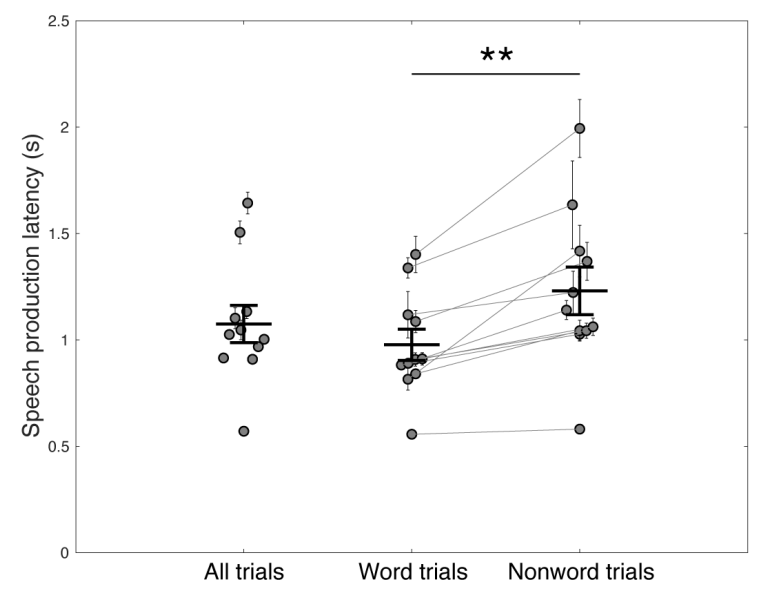

b

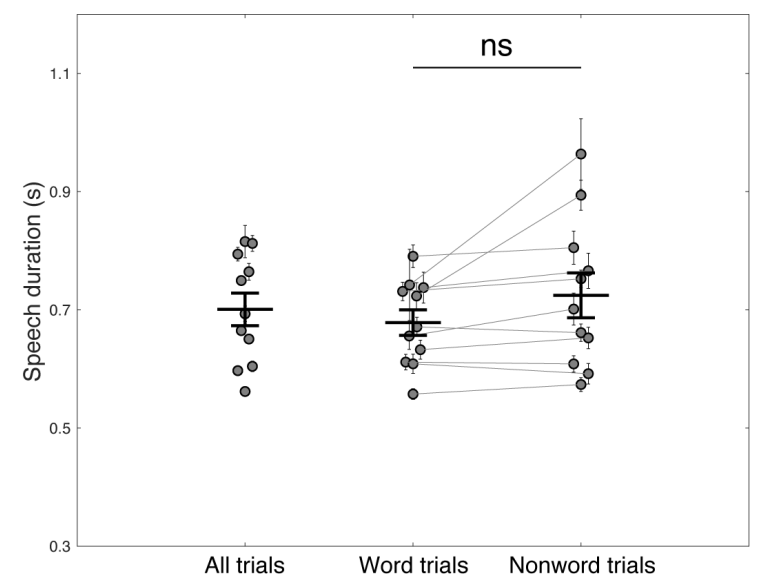


Figure 3: Behavioral outcomes. a Mean and SEM of speech production latency for each subject across all trials (first column), word trials (second column), or nonword trials (third column), superimposed with the mean and SEM of speech production latency across subjects. b Mean and SEM of speech duration for each subject across all trials (first column), word trials (second column), or nonword trials (third column), superimposed with the mean and SEM of speech duration across subjects. Two-tailed paired t-test, ${ }^{* *} P<0.01$, ns: not significant.

\section{Thalamic neural activity is modulated during reading aloud}

Thalamic LFP activity exhibited significant time-frequency modulation during the reading aloud task (Figure 4). Compared to baseline (a period of $1000 \mathrm{~ms}$ preceding stimulus presentation), there was a significant decrease in spectral power in the beta frequency band $(12-30 \mathrm{~Hz})$ that occurred at stimulus presentation and lasted until the end of speech $(-1.08-0.62 \mathrm{~s}$ relative to speech onset, two-tailed Wilcoxon signed-rank test, $n=117, P<0.05$, Bonferroni corrected). In contrast, a significant increase in broadband gamma $(70-150 \mathrm{~Hz})$ activity occurred shortly before the onset of speech and persisted throughout the utterance $(-0.15-0.59$ s relative to speech onset, two-tailed Wilcoxon signed-rank test, $n=117, P<0.05$, Bonferroni corrected). Average $z-$ scored task-related beta and broadband gamma response amplitudes of each trial were then calculated over the respective significant time windows for all the recordings. As a result, 66/117 $(56 \%)$ of the recordings showed significant beta activity decreases during the task compared to baseline (one-tailed one-sample t-test, $P<0.05$, Bonferroni corrected), and significant taskrelated broadband gamma activity increases were observed in 91/117 (78\%) of the recordings (one-tailed one-sample t-test, $P<0.05$, Bonferroni corrected).

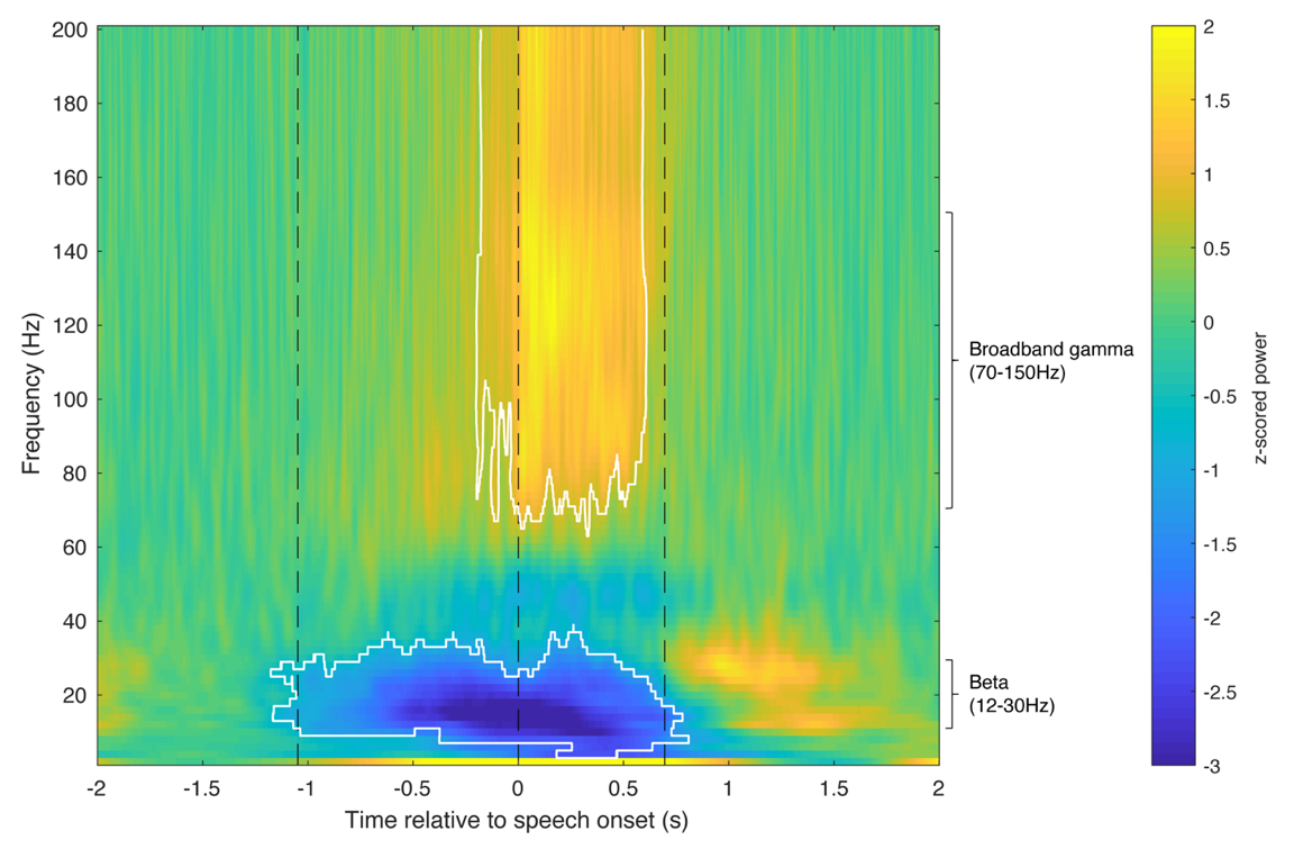


Figure 4: Thalamic neural activity shows task-related modulations. A spectrogram of thalamic neural activity during the reading aloud task, averaged across all trials and all recordings. Trials are aligned to speech onset. Significant changes compared to baseline are marked in white contours (-1.08-0.62s for beta activity, and $-0.15-0.59$ s for broadband gamma activity; Wilcoxon signed-rank test, $n=117, P<0.05$, Bonferroni corrected). Average time points of stimulus presentation, speech onset and offset are marked with black dashed lines.

\section{Beta and broadband gamma responses differ in timing properties}

109 The average interval between stimulus presentation and onset of a significant change from 110 baseline in the spectral power of a particular frequency band, i.e. the mean band response 111 latency, was shorter for significant beta decrease responses than for significant broadband 112 gamma increase responses $(0.79 \pm 0.18 \mathrm{~s}$ vs. $0.99 \pm 0.18 \mathrm{~s}$, two-tailed two-sample $\mathrm{t}$-test, $\mathrm{t}(155)=-$

$\left.1136.97, P<10^{-5}\right)$. The mean band response onset to speech onset interval was also greater for 114 significant beta decrease responses than for significant broadband gamma increase responses

$115\left(0.31 \pm 0.15\right.$ s vs. $0.13 \pm 0.14$ s, two-tailed two-sample $t$-test, $\left.t(155)=7.67, P<10^{-5}\right)$. To characterize

116 the temporal properties of these responses, we examined their trial-to-trial relationships to

117 stimulus presentation versus speech onset (Figure 5a-d). Of the 66 recordings that showed

118 significant task-related beta power decreases, $43(65.2 \%)$ had beta responses time-locked to

119 stimulus presentation, whereas only $19(28.8 \%)$ had beta responses time-locked to speech

120 onset (Figure 5e). In contrast, the majority (70/91, 77.0\%) of significant broadband gamma

121 power increases were time-locked to speech onset, with a minority $(19 / 91,21.0 \%)$ time-locked

122 to stimulus presentation (Figure $5 f$ ). These relationships were dissociated ( $X^{2}$ test, $\alpha=0.05$;

123 Supplementary Table 1$)$, with beta decreases more likely to be stimulus-locked $\left(x^{2}(1)=31.4\right.$,

$\left.124 P<10^{-5}\right)$ and broadband gamma increases more likely to be speech onset-locked $\left(X^{2}(1)=36.1\right.$,

$\left.125 P<10^{-5}\right)$. These temporal correlations did not differ between recording sides $\left(X^{2}\right.$ test, $\alpha=0.05$;

126 Supplementary Table 2). 
a

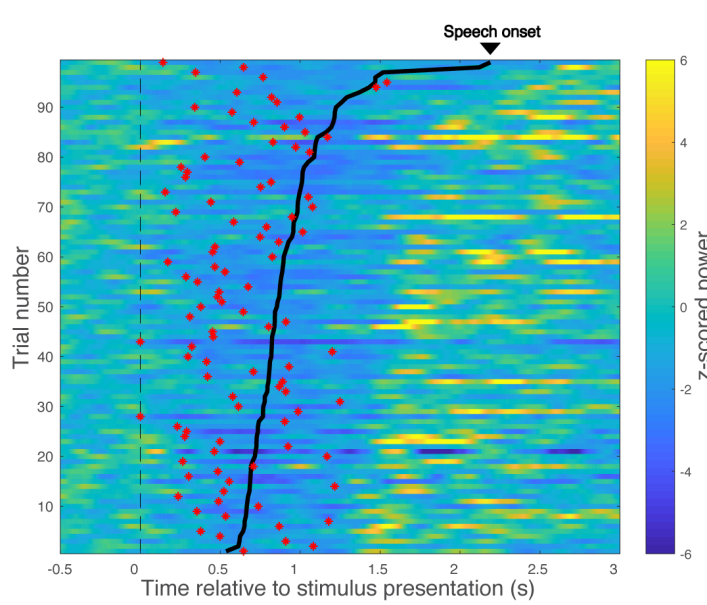

C

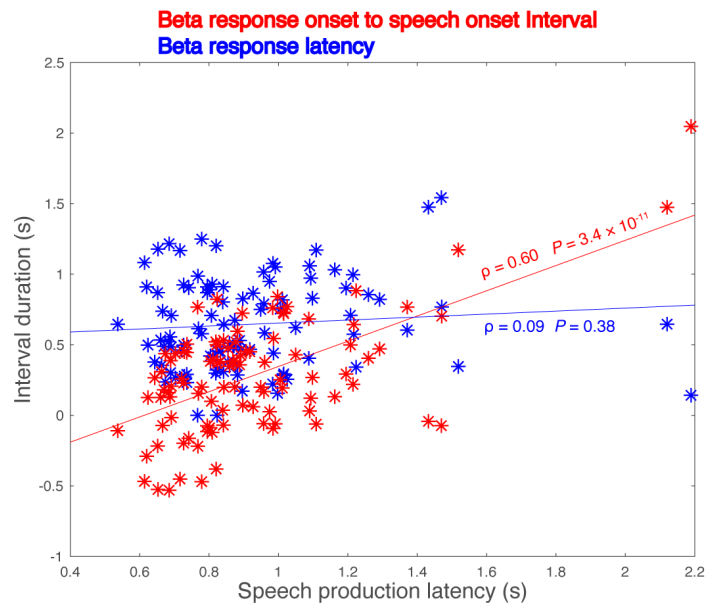

e

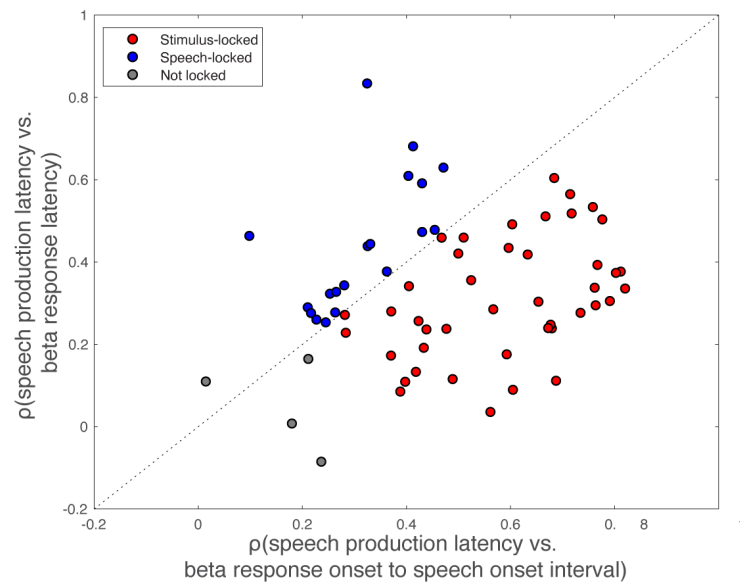

b

Broadband gamma

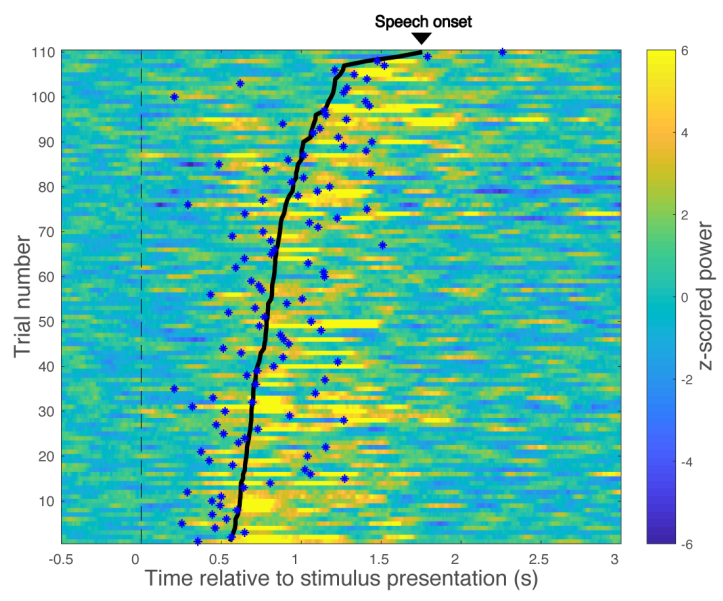

d

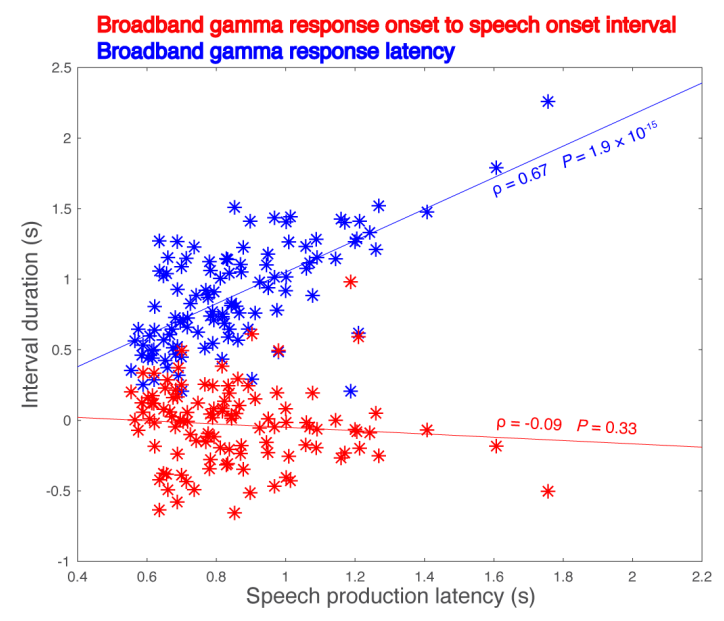

f



Figure 5: Beta decrease response is locked to stimulus presentation while broadband gamma increase response is locked to speech onset. a, b Raster plots for beta band responses (a) and broadband gamma responses (b) across trials of two representative recordings. Trials are aligned to stimulus presentation (indicated with black dashed lines) and sorted by speech production latency. Speech onsets are denoted with bold black lines. Onsets of significant beta activity decreases are marked with red asterisks in a and onsets of significant 
broadband gamma activity increases are marked with blue asterisks in $\mathbf{b} . \mathbf{c}, \mathbf{d}$ Band response onset to speech onset interval (red asterisks) and band response latency (blue asterisks) are correlated (Pearson's correlation, $\alpha=0.05$ ) with speech production latency respectively, for the two representative recordings. $\mathbf{e}, \mathbf{f}$ The same correlation analysis is performed for all the recordings with significant beta decrease responses and all the recordings with significant broadband gamma increase responses, and the results are summarized in $\mathbf{e}$ and $\mathbf{f}$, respectively. Recordings locked to stimulus presentation are shown in red, recordings locked to speech onset are shown in blue, and recordings not locked to either stimulus presentation or speech onset are shown in gray.

\section{Thalamic beta activity is selective to lexical status}

To investigate the involvement and lateralization of the thalamus in lexical processing, only recordings that were from subjects with bilateral lead recordings $(n=7)$ and that showed significant task-related modulation were included for lexicality-related analyses. As a result, 55 recordings (21 unilateral session left-side recordings, 34 from bilateral session left-side (20) and right-side (14) recordings) were included for beta lexical selectivity analysis. Nonword production was associated with a greater suppression of beta power compared with reading words. These differential beta responses were observed in both hemispheres (Figure 6a, c). In the left thalamus, significant word vs. nonword beta amplitude differences occurred between $0.8 \mathrm{~s}$ and $1.8 \mathrm{~s}$ after stimulus presentation (two-tailed paired t-test, $\mathrm{n}=41, P<0.05$, Bonferroni corrected). Similarly, significant beta amplitude differences occurred in the right thalamus at 0.2$1.7 \mathrm{~s}$ relative to stimulus presentation (two-tailed paired t-test, $\mathrm{n}=14, P<0.05$, Bonferroni corrected).

To quantify the lexical selectivity of thalamic beta activity, a lexical selectivity index was determined for each of these recordings. We utilized the time window of significant task-related beta activity decrease (-1.08-0.62s relative to speech onset (see Figure 4)) to calculate a mean task-related beta response power value for each trial, and then the values were compared between nonword trials vs. word trials in each recording by performing a two-sample t-test. The resulting $\mathrm{t}$-statistic was the beta lexical selectivity index for this recording: a beta lexical selectivity index less than zero indicated stronger beta activity decrease (thus having more negative z-scored power) during nonword trials than during word trials, and vice versa. The mean beta lexical selectivity indexes for unilateral session left-side recordings $(-0.49 \pm 0.76)$, bilateral session left-side $(-0.52 \pm 0.82)$ and right-side $(-0.82 \pm 0.89)$ recordings were all significantly less than zero (two-tailed one-sample t-test, $\mathrm{t}(20)=-2.97, P=0.0075 ; \mathrm{t}(19)=-2.83$, $P=0.011 ; \mathrm{t}(13)=-3.48, P=0.0041$; Figure $6 \mathrm{e})$, suggesting that the magnitude of the beta 
170

171

172

173

174

175

176

177

178

179

180

181

182

183

184

185

186

187

188

189

190

191

192

193

194

195

196

197

198

199

200

201

202

203

decrease was significantly nonword-selective in each case. There were no significant differences in beta lexical selectivity between recordings in the left and right thalamus, or between recordings from the unilateral and bilateral sessions on the left side (two-tailed twosample t-test, unilateral session left-side recordings vs. bilateral session right-side recordings: $\mathrm{t}(33)=1.18, P=0.25$; bilateral session left-side recordings vs. bilateral session right-side recordings: $\mathrm{t}(32)=1.05, P=0.30$; unilateral session left-side recordings vs. bilateral session leftside recordings: $\mathrm{t}(39)=0.093, P=0.93)$.

\section{Lexical selectivity of thalamic broadband gamma activity is lateralized}

74 recordings (26 unilateral session left-side recordings, 22 bilateral session left-side recordings, and 26 bilateral session right-side recordings) were included for broadband gamma lexical selectivity analysis. Significantly greater broadband gamma activity increases during nonword production were observed in the left thalamus, starting $0.1 \mathrm{~s}$ after speech onset and persisting throughout the following $0.4 \mathrm{~s}$ (two-tailed paired t-test, $\mathrm{n}=48, P<0.05$, Bonferroni corrected; Figure $6 \mathrm{~b}$ ). In the right thalamus, however, the broadband gamma response curves observed during nonword and word reading were similar, without a significant difference in response amplitudes (two-tailed paired t-test, $n=26, \alpha=0.05$, Bonferroni corrected; Figure 6d).

We next calculated broadband gamma lexical selectivity indexes, using the significant time window of $-0.15-0.59$ s relative to speech onset (see Figure 4). A broadband gamma lexical selectivity index greater than zero indicated stronger broadband gamma activity increase during nonword trials than during word trials, and vice versa. The mean broadband gamma lexical selectivity indexes of both unilateral session left-side recordings $(0.53 \pm 0.96)$ and bilateral session left-side recordings $(0.46 \pm 1.00)$ were significantly greater than zero (two-tailed onesample t-test, $\mathrm{t}(25)=2.80, P=0.0097 ; \mathrm{t}(21)=2.14, P=0.044)$, indicating significant correlation of the magnitude of the gamma response to lexical status (Figure 6f). In contrast, broadband gamma responses in right side recordings did not show significant lexical selectivity $(-0.22$ \pm 0.68 , two-tailed one-sample t-test, $\mathrm{t}(25)=-1.67, P=0.11)$. The differences in broadband gamma lexical selectivity between the left and right thalamus were demonstrated with two-tailed twosample t-test (unilateral session left-side recordings vs. bilateral session right-side recordings: $\mathrm{t}(50)=3.25, P=0.0021$; bilateral session left-side recordings vs. bilateral session right-side recordings: $\mathrm{t}(46)=2.79, P=0.0077)$, further suggesting that selectivity of thalamic broadband gamma activity to lexical status is lateralized to the left. Recordings in the unilateral and bilateral sessions on the left side did not differ in broadband gamma lexical selectivity (two-tailed two- 

selectivity between task sessions.

a

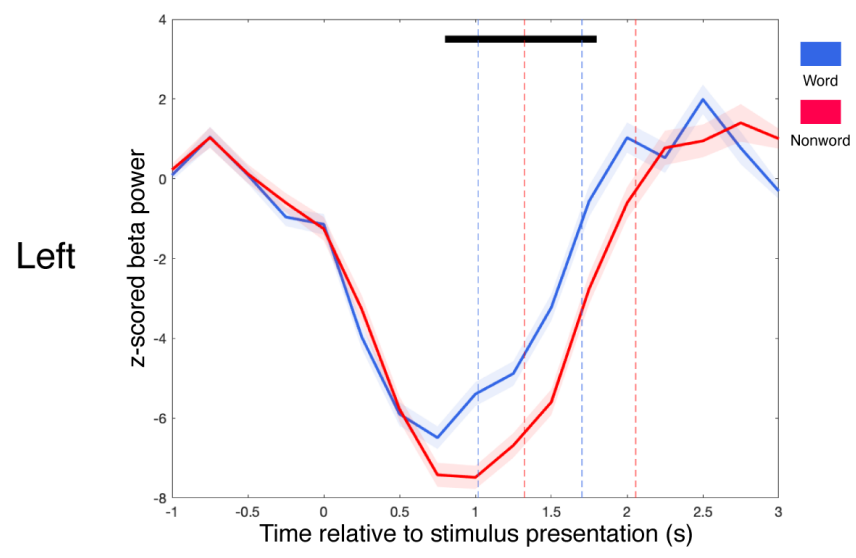

c

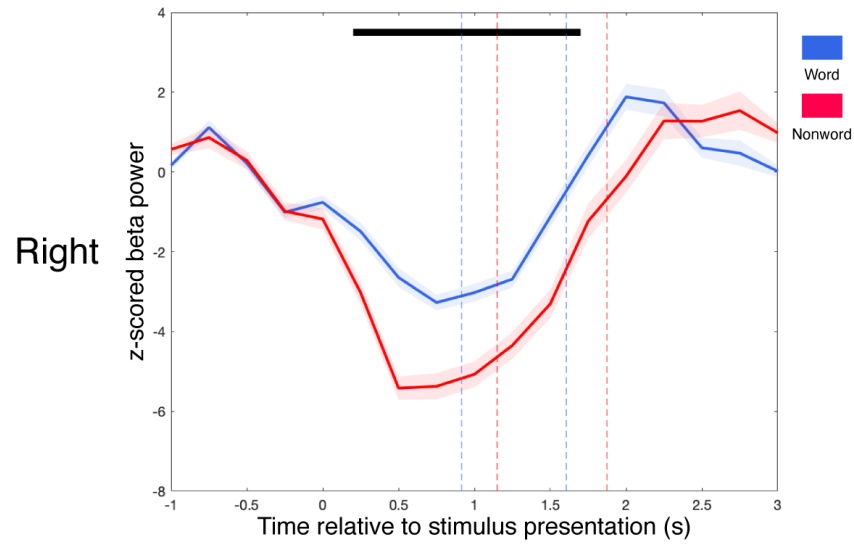

e b



d

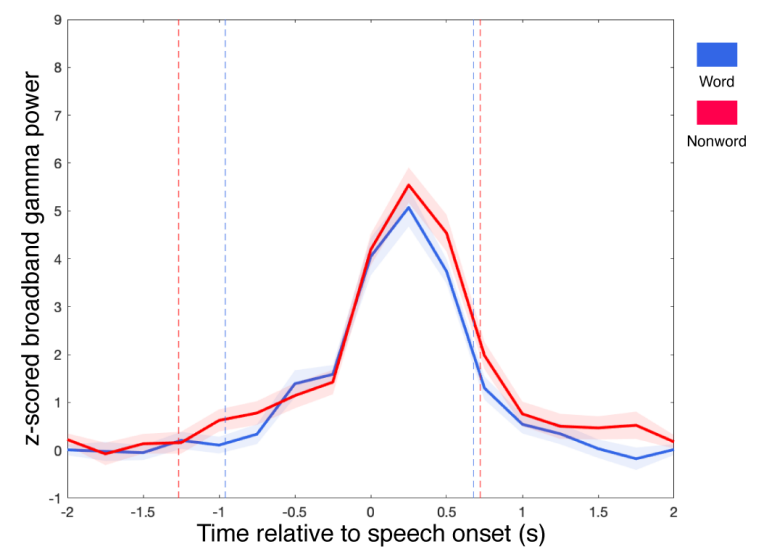

f

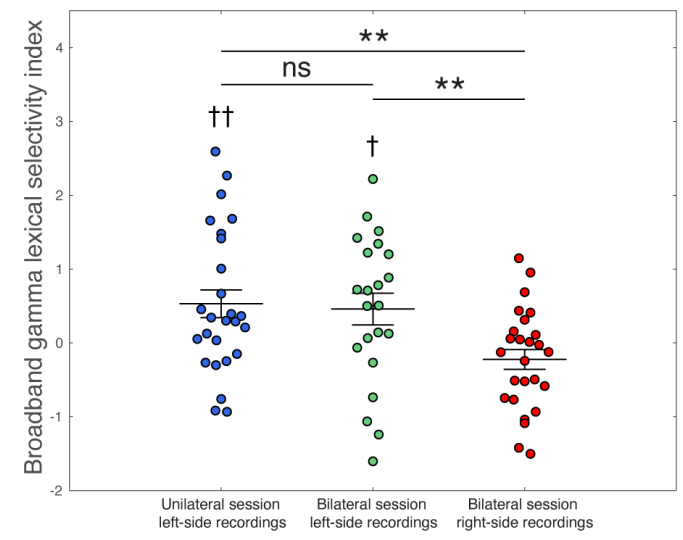

Figure 6: Differential band responses during word vs. nonword reading aloud in the left and right thalamus. a-d Comparisons of average band response amplitudes during word (blue) vs. nonword (red) reading aloud for beta band (a, c) and broadband gamma (b, d), in the left (a, b) and right thalamus (c, d). Band responses are averaged across trials of respective trial types and across recordings that showed significant task-related band responses in each side, aligned 
212 to stimulus presentation for beta band (a, c) and speech onset for broadband gamma (b, d).

213 Average time points of speech onset and end of speech are marked with dashed lines in a and

214 c, and average time points of stimulus presentation and end of speech are marked with dashed

215 lines in $\mathbf{b}$ and $\mathbf{d}$, for word trials (blue) and nonword trials (red) respectively. Black bars indicate

216 significant differences of response amplitudes between word and nonword trials (two-tailed

217 paired t-test, $P<0.05$, Bonferroni corrected). Standard errors are shaded in light colors. e Dot

218 plot of beta lexical selectivity indexes of lead recordings that showed significant task-related

219 beta decrease responses, grouped by recording sides and recording sessions (unilateral

220 session left-side recordings in the first column, bilateral session left-side recordings in the second column, and bilateral session right-side recordings in the third column). Mean and SEM of beta lexical selectivity index across recordings of each group is superimposed on each column, respectively. $f$ Dot plot of broadband gamma lexical selectivity indexes of lead recordings that had significant task-related broadband gamma increase responses, grouped by recording sides and recording sessions (unilateral session left-side recordings in the first column, bilateral session left-side recordings in the second column, and bilateral session rightside recordings in the third column). Mean and SEM of broadband gamma lexical selectivity index across recordings of each group is superimposed on each column, respectively. Twotailed one-sample t-test, $\uparrow P<0.05, \dagger \uparrow P<0.01$; two-tailed two-sample t-test, ${ }^{* *} P<0.01$, ns: not significant.

\section{Left side broadband gamma lexical selectivity is topographically organized}

233 We observed that the recording sites in the left thalamus that were significantly nonword-

234 selective in terms of broadband gamma response activity (broadband gamma lexical selectivity

235 index>1.645 based on normal approximation of t-distribution) appeared to comprise more

236 anterior locations in MNI space (Figure 7a). Pearson's correlation tests demonstrated that the

237 broadband gamma lexical selectivity index significantly correlated with recording location along

238 the anterior-posterior axis (MNI-defined y coordinate; $n=48, \rho=0.49, P=0.00041$; Figure $7 b$ ) and

239 the ventral-dorsal axis (MNI-defined $z$ coordinate; $n=48, \rho=0.30, P=0.041$; Supplementary

240 Figure 1a), but not the lateral-medial axis (MNI-defined $x$ coordinate; $\mathrm{n}=48, \rho=-0.14, P=0.34$;

241 Supplementary Figure 1b) within the left thalamus. To avoid multicollinearity and to test out

242 possible interactions between variables, a stepwise linear regression model was applied, which

243 determined that the anterior-posterior location of the recording was the only significant predictor

244 (estimated coefficient=0.17, $S E=0.044, P=0.00041$ ), while neither the ventral-dorsal location

$245(P=0.72)$ nor the interaction between ventral-dorsal location and anterior-posterior location

$246(P=0.14)$ explained the result. In order to control for subject differences and session differences,

247 we further fitted linear mixed effects models to the data, entering subject and session as random

248 effects. The results indicate that even after accounting for subject and session variability, the

249 broadband gamma lexical selectivity index in the left thalamus had a significant gradient along 
the anterior-posterior axis, with greater broadband gamma lexical selectivity index more likely to be observed anteriorly (estimated coefficient $=0.17$, SE=0.043, $P=0.00032$ ). Based on the linear

252 mixed effects modeling results, neither ventral-dorsal location (estimated coefficient $=0.061$,

253 $\mathrm{SE}=0.035, P=0.087$ ) nor lateral-medial location (estimated coefficient=-0.11, $\mathrm{SE}=0.093, P=0.24$ )

254 of the recording had significant effect on broadband gamma lexical selectivity in the left

255 thalamus. Taken together, these results suggest that broadband gamma lexical selectivity is 256 dependent on the anterior-posterior location of the recording in the left ventral lateral thalamus, 257 with greater nonword selectivity more likely to occur anteriorly.

a

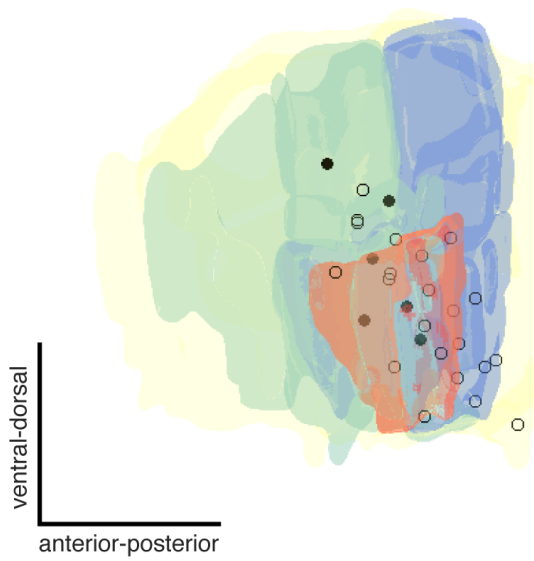

b

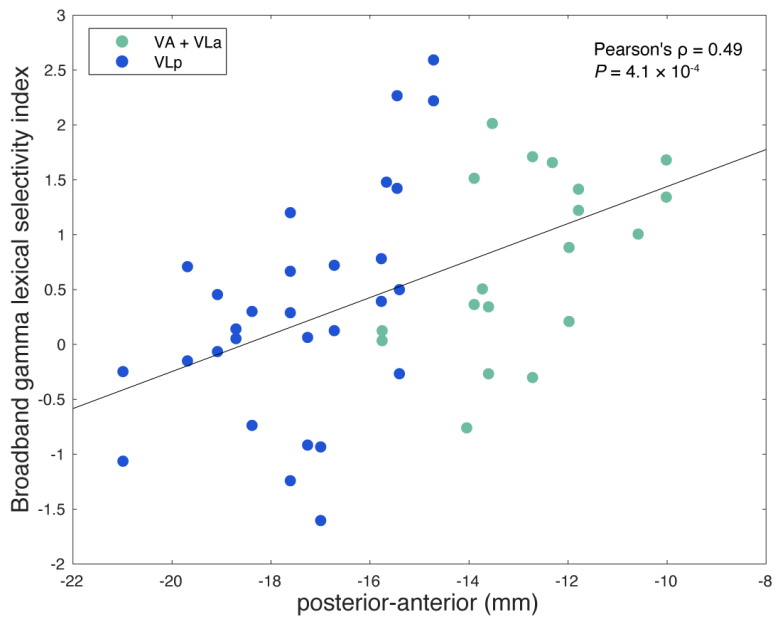

258

259

260

261

262

263

264

265

266

267

268

269

270

271 No significant correlation (Pearson's correlation, $\alpha=0.05$ ) between broadband gamma lexical

272 selectivity index and recording location was observed for right side recordings (Supplementary

273 Figure 2 and 3), or for beta lexical selectivity on either side (Supplementary Figure 4 and 5), by

274 any statistical modeling. These results suggest that the topography of lexical selectivity is

275 unique to broadband gamma responses in the left thalamus.

Figure 7: Broadband gamma lexical selectivity depends on anterior-posterior location of the recording in the left thalamus. a Left side lead recording sites are plotted together with anatomical structures (thalamus in yellow, VA and VLa in green, VLp in blue, and Vim in orange), viewed from a lateral direction. Recording sites where significantly nonword-selective recordings in terms of broadband gamma response amplitudes (broadband gamma lexical selectivity index>1.645 based on normal approximation of t-distribution) were observed in either session are filled with black, and remaining recording sites are shown in open circles. $\mathbf{b}$ Broadband gamma lexical selectivity indexes of left side lead recordings that showed significant task-related broadband gamma responses are correlated (Pearson's correlation, $\alpha=0.05$ ) with recording locations along the anterior-posterior axis (y coordinates in MNI space). Recordings inside VA or VLa are green-colored and recordings inside VLp are blue-colored. 


\section{Discussion}

278 Subcortical contributions to language production have been hypothesized largely from

279 correlations of focal brain damage with aphasic syndromes and from language studies using

280 functional magnetic resonance imaging (fMRI) or scalp electroencephalography (EEG) (Hebb \&

281 Ojemann, 2013). Our results are the first to demonstrate thalamic neural activity during reading

282 aloud. We discovered that the encoding of lexical status is differentially represented in thalamic

283 neural activity. Whereas greater beta activity decrease occurred during nonword trials as

284 compared to word trials in both hemispheres, greater gamma increases in the left but not right

285 thalamus were associated with the spoken production of nonwords as compared to words.

286 Importantly, the level of broadband gamma lexical selectivity was greater in more anterior

287 thalamic recording locations, regions more likely to receive basal ganglia than cerebellar

288 afferents (Alexander et al., 1986).

We first observed that thalamic beta activity showed a task-related decrease, locked to stimulus presentation, consistent with the expected beta desynchronization that accompanies motor action. Beta oscillations are proposed to signal the maintenance of current sensorimotor and cognitive states (Engel \& Fries, 2010), and event-related beta activity decreases during the preparation and the execution of voluntary movements have been observed across relevant brain regions, including the thalamus (Klostermann et al., 2007; Kühn et al., 2004; Paradiso, 2004; Tzagarakis et al., 2010). Beta activity was reduced across the entirety of each task event, which requires a series of underlying neural dynamics (processing the visual stimulus, retrieval of lexical information, encoding of the phonological information, and execution of articulatory movement), suggesting a state change that facilitated the process of speech production. to speech onset. Given that broadband gamma activity is thought to index synchronized local neuronal firing (Ray et al., 2008; Ray \& Maunsell, 2011) and is associated with the functional activation of relevant brain regions during a wide range of sensorimotor and cognitive tasks, including speech and language processing (Bouchard et al., 2013; Chrabaszcz et al., 2019; Crone et al., 2006; Juphard et al., 2011; Uhlhaas et al., 2011), our data suggest that the ventral lateral thalamus tracks speech production. confined to cortical regions (Dietz et al., 2005; Fiez et al., 1999; Hagoort et al., 1999; Heim et 
311 study, we utilized the balanced design of words and nonwords in the stimulus sets and

312 simultaneous bilateral recordings in the seven participants to determine whether lexical

313 processing is differentially represented in the thalamus during reading aloud. Thalamic

314 broadband gamma oscillations showed significantly stronger activation during nonword reading

315 aloud than during word reading aloud, which evolved around speech onset and was present

316 throughout the entire utterance. Notably, this broadband gamma lexical selectivity was found

317 only in recordings from the left side. According to classic speech production models (Levelt et

318 al., 1999), the time course of the observed broadband gamma activity difference between word

319 and nonword trials corresponded to the articulatory stage of reading aloud. It is unlikely that this

320 lexicality effect was motoric, considering that the motor complexity was balanced between word

321 and nonword stimuli, and that this differential broadband gamma modulation was not

322 equivalently observed in the right hemisphere. In fact, the dual-route theory of reading aloud has

323 suggested that words and nonwords are read aloud differently: while real words can be read

324 aloud via either grapheme-to-phoneme conversions or direct word-to-sound mapping, nonwords can only be read aloud via grapheme-to-phoneme conversions (Coltheart et al., 2001). It is possible that phonological encoding was still ongoing during nonword production, after a failed internal lexicon lookup procedure. Indeed, stronger gamma $(50-150 \mathrm{~Hz})$ responses for pseudowords than for words have been reported in Broca's area (Brodmann's areas 44 and 45) during silent word reading, where the length of this differential response increased with the length of the stimuli (Juphard et al., 2011). In addition, it is possible that reading aloud nonwords created a learning or error correction demand (Hickok, 2014). We propose that the lexicality effect on thalamic broadband gamma activity during reading may reflect left thalamic participation in

333 phonological encoding, learning, and feedback monitoring during speech and language

334 processing. Although previous studies have supported a lateralized thalamic role in language

335 (Johnson \& Ojemann, 2000), our results from simultaneous bilateral direct recordings in the 336 thalamus are the first to provide direct supporting evidence. lexical selectivity of the broadband gamma response was significantly correlated with the 340 anterior-posterior location of recording sites in the left thalamus, with a higher lexical selectivity 341 more likely to appear anteriorly. In contrast, a gradient for broadband gamma lexical selectivity 342 was not observed in the right thalamus. This finding further supports a unique language role 343 lateralized to the left thalamus, and suggests functional heterogeneity in the left ventral lateral 344 thalamus during speech and language processing. The anterior portion (VA and VLa) receives 
input primarily from the internal globus pallidus and substantia nigra pars reticulata, and has strong connections with frontal cortex, including Broca's area (Alexander et al., 1986; Behrens et al., 2003; Bosch-Bouju et al., 2013; Hintzen et al., 2018; Hwang et al., 2017; Zhang et al., 2010). Broca's area has consistently been associated with lexico-phonological processing (Dietz et al., 2005; Fiez et al., 1999; Hagoort et al., 1999; Heim et al., 2005; Herbster et al., 1997; Juphard et al., 2011; Mechelli et al., 2003; Xu, 2001). Thus, this thalamic region may participate in differential lexical processing during nonword vs. word reading aloud in concert with Broca's area. In contrast, the posterior region (VLp), which receives cerebellar projections and preferentially sends output to primary motor cortex (Behrens et al., 2003; Bosch-Bouju et al., 2013; Hintzen et al., 2018; Hwang et al., 2017; Zhang et al., 2010), might be more related to motor control of a selected motor plan. This idea is supported by previous stimulation studies that have reported location-dependent effects of thalamic stimulation on speech and language: stimulation of VLa could cause acceleration of language processes, while stimulation of VLp often affected motor aspects of speech, such as perseveration and stuttering speech (Hebb \& Ojemann, 2013). We note that VA has been proposed to participate in selection of a language unit during speech production, via basal ganglia-thalamo-cortical loop interactions (Crosson, 2013); it also is included in the "planning loop" in the GODIVA model of speech production (Bohland et al., 2010).

In contrast, although significantly stronger nonword-related than word-related beta activity decreases accompanied the reading aloud task bilaterally, neither lateralization nor topography was observed for thalamic beta lexical selectivity. Therefore, beta activity decreases likely represent nonspecific changes of cognitive and sensorimotor states that prepare the entire thalamo-cortical network for a behavioral change. Note that in the current work we did not try to compare task-related band response strength between different recording locations and different recording sides among subjects and sessions, because of a number of uncontrollable factors that affect the band oscillatory power (e.g. recording impedances and baseline neural activity are variable across subjects, sessions, and recording sides). In contrast, the lexical selectivity index, which was calculated by comparing word-related and nonword-related band response strength within each recording, is mostly independent of those factors and thus comparable at the group level. This idea is supported by the fact that lexical selectivity indexes in both beta and broadband gamma bands remained consistent across sessions. 
378 In summary, our results are the first demonstration of time-frequency modulations of thalamic

379 neural activity during reading aloud. These data suggest that lateralized and topographically

380 organized thalamic pathways participate in speech production differently, based on whether a

381 word or nonword is being read.

382

\section{Materials and methods}

\section{Subjects}

385 Eleven human subjects (3 females, $68.4 \pm 8.0$ years) with essential tremor undergoing awake 386 DBS implantation surgery targeting the Vim nucleus of the thalamus were studied. All 387 participants were right-handed native English speakers. None had significant cognitive 388 impairment based on a detailed neuropsychological evaluation performed during clinical 389 evaluation for DBS surgery. All but one underwent bilateral (left side first) DBS lead implantation 390 (one subject had one lead implanted in the left hemisphere previously and underwent right side 391 lead implantation in the current study). Full demographic description of subjects is provided in 392 Table 1. All protocols were approved by the Institutional Review Board of the University of 393 Pittsburgh (IRB Protocol \#PRO13110420), and all participants gave written informed consent. 
Table 1: Subject characteristics.

\begin{tabular}{|c|c|c|c|c|c|c|c|c|c|}
\hline Subject & Age & Gender & Handedness & MMSE & $\begin{array}{c}\text { LFP } \\
\text { recording } \\
\text { side }\end{array}$ & $\begin{array}{c}\text { LFP } \\
\text { recording } \\
\text { type }\end{array}$ & $\begin{array}{c}\text { Number of } \\
\text { recordings }\end{array}$ & $\begin{array}{c}\text { Mean } \\
\text { number of } \\
\text { trials per } \\
\text { session }\end{array}$ & $\begin{array}{c}\text { Trials } \\
\text { rejected, } \\
\%\end{array}$ \\
\hline 1 & 61 & Male & Right & NR & Left & $\begin{array}{c}\text { Mapping } \\
\text { electrodes }\end{array}$ & 12 & 111 & 7 \\
\hline 2 & 70 & Female & Right & $30 / 30$ & Right & $\begin{array}{c}\text { Mapping } \\
\text { electrodes }\end{array}$ & 6 & 107 & 11 \\
\hline 3 & 66 & Male & Right & NR & Left & $\begin{array}{c}\text { Mapping } \\
\text { electrodes }\end{array}$ & 6 & 116 & 3 \\
\hline 4 & 75 & Male & Right & $26 / 30$ & Left & $\begin{array}{c}\text { Mapping } \\
\text { electrodes }\end{array}$ & 9 & 112 & 5 \\
\hline 5 & 64 & Male & Right & $30 / 30$ & Both & DBS leads & 12 & 104 & 13 \\
\hline 6 & 53 & Male & Right & NR & Both & DBS leads & 12 & 113 & 5 \\
\hline 7 & 67 & Male & Right & $26 / 30$ & Both & DBS leads & 12 & 111 & 7 \\
\hline 8 & 71 & Male & Right & $26 / 30$ & Both & DBS leads & 12 & 108 & 9 \\
\hline 9 & 84 & Female & Right & $30 / 30$ & Both & DBS leads & 12 & 118 & 2 \\
\hline 10 & 73 & Female & Right & $30 / 30$ & Both & DBS leads & 12 & 109 & 9 \\
\hline 11 & 68 & Male & Right & $28 / 30$ & Both & DBS leads & 12 & 114 & 5 \\
\hline
\end{tabular}

MMSE: Mini-Mental State Examination; NR: not recorded.

\section{Stimuli and experimental paradigm}

398 Subjects performed a reading aloud task either in the course of subcortical mapping procedure during the surgery (4/11 subjects with mapping electrode recordings) or after the placement of DBS lead in each side (7/11 subjects with lead electrode recordings). For the 4 subjects with mapping electrode recordings, each subject performed up to 4 task sessions, while the 7 subjects with lead electrode recordings each performed 2 task sessions (the first session occurred after left lead was implanted, and the second session took place after bilateral implantation was completed). Each session included 120 trials. The stimuli were consonantvowel-consonant (CVC) words and nonwords that were presented on a computer screen. Four lists of 120 stimuli (Supplementary Table 3) were constructed based on a previous work (Moore et al., 2017). The first 60 stimuli of each list alternated between unique words and nonwords, and those words and nonwords were balanced along a number of psycholinguistic features, including phoneme probability, phonological neighborhood density, bigram frequency, and 
410 biphone probability (see Supplementary Table 4 for the results of statistical comparisons (two-

411 tailed two-sample t-test, $n=60, \alpha=0.05$ ) between the two conditions in terms of these

412 psycholinguistic parameters, which were calculated using the English Lexicon Project database

413 (Balota et al., 2007)). The nonwords were duplicated twice to construct the last 60 stimuli of

414 each list. One of the four stimulus lists was presented to the subjects during each task session.

415 For lexicality-related analyses, only the first 60 trials of each session were used.

417 The experimental paradigm was programmed using MATLAB (MathWorks, Natick, MA) and

418 Psychophysics Toolbox extensions (Brainard, 1997). A schematic of the experiment is shown in

419 Figure 1. Before each trial, a white fixation cross was presented on the screen. Each trial was

420 initiated manually by the experimenter, with the appearance of a green fixation cross on the

421 screen. The green fixation cross lasted $250 \mathrm{~ms}$, and was followed by a variable time delay (500-

$4221000 \mathrm{~ms}$ ) during which the screen remained black. Then the CVC syllable stimulus was

423 presented in white on the screen, and subjects were instructed to read it aloud. The text

424 remained on the screen until the subjects finished speaking. A white fixation cross was

425 presented on the screen during the inter-trial interval (ITI).

\section{Electrophysiological recordings}

428 For the 4/11 subjects where physiological subcortical mapping was administered, LFP recordings were performed using the Neuro-Omega recording system (Alpha Omega, Nazareth, Israel) and mapping electrodes that have a stainless steel macroelectrode ring $(0.55 \mathrm{~mm}$ in

431 diameter, $1.4 \mathrm{~mm}$ in length) $3 \mathrm{~mm}$ above the tip, while in the other $7 / 11$ subjects LFP signal was

432 recorded from Medtronic Model 3387 DBS leads (Medtronic, Minneapolis, MN) with four

433 platinum-iridium electrodes $(1.27 \mathrm{~mm}$ in diameter, $1.5 \mathrm{~mm}$ in length) that are spaced $1.5 \mathrm{~mm}$

434 apart, using Grapevine Neural Interface Processor (Ripple LLC, Salt Lake City, UT). The

435 mapping electrodes and the DBS leads targeted the Vim. For subjects with mapping electrode

436 recordings, three mapping electrodes were placed in three trajectories (anterior, central, and

437 posterior or central, posterior, and medial) of a standard cross-shaped Ben-Gun array with a

$4382 \mathrm{~mm}$ center-to-center spacing, and made simultaneous recordings starting at $15 \mathrm{~mm}$ above the

439 surgical target with manual advance in $0.1 \mathrm{~mm}$ steps. The reading aloud task was carried out in

440 pauses during subcortical mapping procedure and subjects performed in up to four recording 441 sessions, with each session corresponding to a different recording depth. Mapping electrode

442 recordings were performed only during the left side implantation except for one subject who

443 underwent unilateral implantation in the right side, and thus got mapping electrode recordings 
only in the right side. For subjects with DBS lead electrode recordings, the task was administered in two recording sessions, one after the implantation of the DBS lead in the left side, receiving recordings from only the left DBS lead electrodes, and the other after bilateral DBS lead implantation was completed, receiving simultaneous recordings from bilateral lead electrodes. The LFP signal recorded from mapping electrodes was sampled at $44 \mathrm{kHz}$ and bandpass filtered from $0.075 \mathrm{~Hz}$ to $10 \mathrm{kHz}$, and data recorded from DBS lead electrodes were collected at $30 \mathrm{kHz}$. Signal collected in one recording site in one session counted as one recording. Subject recording characteristics are summarized in Table 1.

\section{Audio recordings}

Subjects' speech signal was recorded using an omnidirectional microphone (Audio-Technica model ATR3350iS Mic, frequency response 50-18,000Hz (Audio-Technica, Machida, Japan) for 6 subjects, and PreSonus model PRM1 Precision Flat Frequency Mic, frequency response 20$20,000 \mathrm{~Hz}$ (PreSonus, Baton Rouge, LA) for 5 subjects) placed approximately $8 \mathrm{~cm}$ away from the subject's mouth and oriented at an angle of about $45^{\circ}$. The audio signal was collected by Grapevine Neural Interface Processor at a sampling frequency of $30 \mathrm{kHz}$. For subjects with mapping electrode recordings, the audio signal was then synchronized with neural signal recorded by the Neuro-Omega system using digital pulses delivered to both recording systems via a USB data acquisition unit (model USB-1208FS, Measurement Computing, Norton, MA).

\section{Electrode localization}

DBS lead electrodes and mapping electrodes were localized using LEAD-DBS toolbox (Horn et al., 2019; Horn \& Kühn, 2015). Postoperative brain scans were coregistered to preoperative brain scans using open-source Advanced Normalization Tools (ANTs). Pre- and postoperative acquisitions were then normalized into MNI ICBM152 NLIN 2009b stereotactic space (Fonov et al., 2011). Both coregistration and normalization results underwent manual quality check. Semiautomatic reconstruction of electrodes in MNI space was performed in LEAD-DBS and MNIdefined coordinates were determined for all the electrode contacts (Figure 2). A digitized and normalized to MNI space version of the Ewert atlas (Ewert et al., 2018) was used to categorize the electrode contacts. A contact was assigned to a nucleus if it was within or in the vicinity of the nucleus ( $1 \mathrm{~mm}$ cut-off) based on the minimum Euclidean distance between the contact and the voxels of the nucleus.

\section{Data pre-processing}


478 The audio signal was segmented into trials and the speech sound was coded by communication science students trained in phonetics in a custom-designed graphical user interface implemented in MATLAB. The coding results were manually checked by a speech-language pathologist. For each trial, (1) the onset of speech was identified, (2) the end of speech was identified, and (3) the speech content was identified. Trials were considered to have correct speech responses and were included in further analyses if they met all the following criteria: (1) the subject's speech response could be clearly identified by the coder, (2) the subject's response was a CVC syllable consisting of the targeted phonemes, and (3) the response did not make nonword into a word or word into a nonword.

Electrophysiological data were pre-processed using custom code based on FieldTrip toolbox (Oostenveld et al., 2011) in MATLAB. The data were resampled at $1 \mathrm{kHz}$ and band-pass filtered from 2 to $400 \mathrm{~Hz}$. The data were also notch-filtered at $60 \mathrm{~Hz}$ and its' harmonics to remove line noise. Time series data from all recording sites were visually and quantitatively inspected for quality control. The data were then segmented into trials, each spanning $2 \mathrm{~s}$ before stimulus presentation to $2 s$ after the end of speech. Trials with artefacts or excessive noise were identified both manually and quantitatively, and were excluded for subsequent analyses. Combined with trials that did not meet the criteria for correct speech responses, an average 6.9 $\pm 3.4 \%$ of trials per subject were rejected (Table 1 ). The remaining data were common-average referenced to minimize noise. For spectral-temporal analysis, the data were decomposed using Morlet wavelet transformation (width=7) over frequencies of $2 \mathrm{~Hz}$ to $200 \mathrm{~Hz}$ in increment steps of $2 \mathrm{~Hz}$. For band activity analyses, instantaneous analytic amplitudes of beta and broadband gamma frequency bands were extracted from respective bandpass filters using Hilbert transform (MATLAB function hilbert). The resulting signal of each trial was z-scored relative to the baseline (a period of $1000 \mathrm{~ms}$ preceding stimulus presentation).

\section{Task-related responses}

505 Time-frequency data were averaged across all trials centering on speech onset (from $2 \mathrm{~s}$ before

506 speech onset to $2 \mathrm{~s}$ after speech onset), z-scored to baseline, and then averaged across all

507 recordings. Nonparametric two-tailed Wilcoxon signed-rank test was performed to determine

508 significant time-frequency modulations compared to baseline $(n=117, \alpha=0.05$, Bonferroni

509 corrected). Significant time windows for beta and broadband gamma determined by the

510 statistical results were used to calculate a mean beta response strength and mean broadband

511 gamma response strength for each trial in each recording. Then one-tailed one-sample t-test 
512 was performed on each recording to determine recordings that had significant beta activity

513 decreases and recordings that had significant broadband gamma activity increases, respectively

$514(\alpha=0.05$, Bonferroni corrected).

515

516 Locking analysis

517 In an effort to characterize the timing properties of beta decrease response and broadband

518 gamma increase response for recordings with significant task-related changes in either band

519 activity, we examined the trial-to-trial relationships of significant band response onsets to

520 stimulus presentation versus speech onset in these recordings. First, beta or broadband gamma

521 time series data of each trial were smoothed using a moving average kernel of 200ms (MATLAB

522 function smoothdata) and z-scored to baseline, in order to minimize single-trial noise. For

523 recordings with significant beta decrease responses, a thresholding method with a critical value

524 of $z=-1.645$ was applied to determine the onset of beta response for each trial. Specifically, for

525 each trial, the period with band response power below the threshold that gave minimum

526 summed activity was considered as activation period and the beginning of the period was

527 determined as onset of beta response. For recordings with significant broadband gamma

528 increase responses, the onset of broadband gamma increase was determined in a similar way,

529 except that a critical value of $z=1.645$ was used and period of maximum summed activity above

530 the threshold was considered as activation period. For each band, a trial of one recording was

531 discarded for locking analysis if no beyond-threshold period was present throughout the trial.

532 Two intervals were calculated for each trial: (1) the interval between stimulus presentation and

533 the onset of significant band response (band response latency), and (2) the interval between the

534 onset of significant band response and the onset of speech. Then the two intervals were

535 correlated (Pearson's correlation, $\alpha=0.05$ ) with speech production latency (interval between

536 stimulus presentation and onset of speech) across trials for each recording, respectively. The

537 band response of a recording was considered to be more time-locked to stimulus presentation,

538 if (1) band response onset to speech onset interval was significantly correlated with speech

539 production latency, and (2) the correlation coefficient (Pearson's $\rho$ ) between band response

540 onset to speech onset interval and speech production latency was greater than the correlation

541 coefficient between band response latency and speech production latency. On the contrary, the

542 band response was considered to be more time-locked to speech onset, if (1) band response

543 latency was significantly correlated with speech production latency, and (2) the correlation

544 coefficient between band response latency and speech production latency was greater than the

545 correlation coefficient between band response onset to speech onset interval and speech 
546 production latency. If a band response did not meet any of the two criteria, it was considered not

547 locked to either stimulus presentation or speech onset. $x^{2}$ tests were performed to differentiate

548 the locking properties of broadband gamma increase response and beta decrease response,

549 and to test if the locking properties differed between recording sides $(\alpha=0.05)$.

550

551 Lexical selectivity analysis

552 We utilized the first 60 trials of recordings that were from the seven subjects with bilateral data

553 and that showed significant task-related modulations in beta or broadband gamma activity to

554 make comparisons between the left and right thalamus in terms of band lexical selectivity. A

555 mean z-scored band response curve averaged across trials and across recordings was

556 obtained for each frequency band, each trial type (word/nonword), in each side. Trials were

557 aligned to stimulus presentation for beta and aligned to speech onset for broadband gamma,

558 based on their respective locking properties. Periods of significant differences between word-

559 related responses and nonword-related responses were determined with two-tailed paired t-test

560 using a sliding window of $100 \mathrm{~ms}$ ( $\alpha=0.05$, Bonferroni corrected).

562 To quantify the extent of band response difference between word and nonword trials, beta

563 lexical selectivity indexes and broadband gamma lexical selectivity indexes were calculated for

564 respective recordings. For each recording, the mean response power values of a particular

565 frequency band over the corresponding significant time window determined before (see Task-

566 related responses) were calculated for word trials and nonword trials. Two-sample t-test was

567 then performed between nonword-related power values and word-related power values, and the 568 resulting t-statistic was the lexical selectivity index of that frequency band for that recording. For 569 beta, a lexical selectivity index smaller than zero meant that the recording showed stronger beta 570 activity decrease (thus having more negative z-scored power) during nonword trials than during 571 word trials, and vice versa. For broadband gamma, a lexical selectivity index greater than zero

572 indicated that the recording showed stronger broadband gamma activity increase during

573 nonword reading aloud than during word reading aloud, and vice versa. The beta or broadband

574 gamma activity of a recording was considered significantly nonword-selective, if lexical

575 selectivity index was smaller than -1.645 for beta or greater than 1.645 for broadband gamma

576 (normal approximation of t-distribution). Two-tailed one-sample t-test was performed to test if

577 lexical selectivity indexes of a selected group of recordings were significantly different from zero

$578(\alpha=0.05)$. Two-tailed two-sample t-test was performed to test if lexical selectivity indexes

579 significantly differed between two groups of recordings $(\alpha=0.05)$. 


\section{Analysis of location dependency of lexical selectivity}

582 We further sought to examine possible dependence of band lexical selectivity on recording 583 location, for both beta band and broadband gamma and in both left and right recording sides.

584 Several regression models were applied to the data. First, simple linear regression (Pearson's

585 correlation, $\alpha=0.05$ ) was performed to correlate lexical selectivity index with recording location:

586 anterior-posterior location (MNI-defined y coordinate), ventral-dorsal location (MNI-defined z 587 coordinate), and lateral-medial location (MNI-defined x coordinate), respectively. A series of 588 stepwise linear regression models (MATLAB function stepwiselm, response variable: lexical 589 selectivity index, predictor variables: MNI-defined $x, y, z$ coordinates and their interactions, $590 \alpha=0.05)$ were then carried out to test out possible variable interactions, minimize 591 multicollinearity, and determine the final location dependency model of band lexical selectivity.

592 Finally, in order to account for possible lexical selectivity differences that might exist due to 593 subject- and session-specific variations, linear mixed effects models (MATLAB function fitglme) 594 were also applied to the data, setting subject and session as random effects.

\section{Acknowledgments}

597 This work was supported by NIH grant U01NS098969 to R.M.R., the Hamot Health foundation 598 to R.M.R., and a University of Pittsburgh Brain Institute NeuroDiscovery Pilot Research Award 599 to R.M.R. This work was supported by the University of Pittsburgh-Tsinghua University Scholars 600 Program to D.W.

601

\section{Competing interests}

603 The authors declare no competing interests.

References

Alexander, G. E., DeLong, M. R., \& Strick, P. L. (1986). Parallel Organization of Functionally Segregated Circuits Linking Basal Ganglia and Cortex. Annual Review of Neuroscience, 9(1), 357-381. https://doi.org/10.1146/annurev.ne.09.030186.002041 
613

Behrens, T. E. J., Johansen-Berg, H., Woolrich, M. W., Smith, S. M., Wheeler-Kingshott, C. A. M., Boulby, P. A., Barker, G. J., Sillery, E. L., Sheehan, K., Ciccarelli, O., Thompson, A. J., Brady, J. M., \& Matthews, P. M. (2003). Non-invasive mapping of connections between human thalamus and cortex using diffusion imaging. Nature Neuroscience, 6(7), 750-757. https://doi.org/10.1038/nn1075

Bohland, J. W., Bullock, D., \& Guenther, F. H. (2010). Neural representations and mechanisms for the performance of simple speech sequences. Journal of Cognitive Neuroscience, 22(7), 1504-1529. https://doi.org/10.1162/jocn.2009.21306

Bosch-Bouju, C., Hyland, B. I., \& Parr-Brownlie, L. C. (2013). Motor thalamus integration of cortical, cerebellar and basal ganglia information: Implications for normal and parkinsonian conditions. In Frontiers in Computational Neuroscience (Issue NOV). https://doi.org/10.3389/fncom.2013.00163

Bouchard, K. E., Mesgarani, N., Johnson, K., \& Chang, E. F. (2013). Functional organization of human sensorimotor cortex for speech articulation. Nature, 495(7441), 327-332. https://doi.org/10.1038/nature11911

Brainard, D. H. (1997). The Psychophysics Toolbox. Spatial Vision, 10(4), 433-436. http://www.ncbi.nlm.nih.gov/pubmed/9176952

Chrabaszcz, A., Neumann, W. J., Stretcu, O., Lipski, W. J., Bush, A., Dastolfo-Hromack, C. A., Wang, D., Crammond, D. J., Shaiman, S., Dickey, M. W., Holt, L. L., Turner, R. S., Fiez, J. A., \& Richardson, R. M. (2019). Subthalamic nucleus and sensorimotor cortex activity during speech production. Journal of Neuroscience, 39(14), 2698-2708. https://doi.org/10.1523/JNEUROSCI.2842-18.2019

Coltheart, M., Rastle, K., Perry, C., Langdon, R., \& Ziegler, J. (2001). DRC: A dual route cascaded model of visual word recognition and reading aloud. Psychological Review, 108(1), 204-256. https://doi.org/10.1037/0033-295X.108.1.204

Crone, N. E., Sinai, A., \& Korzeniewska, A. (2006). High-frequency gamma oscillations and human brain mapping with electrocorticography. Progress in Brain Research, 159, 275 295. https://doi.org/10.1016/S0079-6123(06)59019-3

Crosson, B. (2013). Thalamic mechanisms in language: a reconsideration based on recent findings and concepts. Brain and Language, 126(1), 73-88. https://doi.org/10.1016/j.bandl.2012.06.011

Dietz, N. A. E., Jones, K. M., Gareau, L., Zeffiro, T. A., \& Eden, G. F. (2005). Phonological decoding involves left posterior fusiform gyrus. Human Brain Mapping, 26(2), 81-93. https://doi.org/10.1002/hbm.20122 
647

648

649

650

651

652

653

654

655

656

657

658

659

660

661

662

663

664

665

666

667

668

669

670

671

672

673

674

675

676

677

678

679

680

Engel, A. K., \& Fries, P. (2010). Beta-band oscillations-signalling the status quo? Current Opinion in Neurobiology, 20(2), 156-165. https://doi.org/10.1016/j.conb.2010.02.015

Ewert, S., Plettig, P., Li, N., Chakravarty, M. M., Collins, D. L., Herrington, T. M., Kühn, A. A., \& Horn, A. (2018). Toward defining deep brain stimulation targets in MNI space: A subcortical atlas based on multimodal MRI, histology and structural connectivity. Neurolmage, 170, 271-282. https://doi.org/10.1016/j.neuroimage.2017.05.015

Fiez, J. A., Balota, D. A., Raichle, M. E., \& Petersent, S. E. (1999). Effects of lexicality, frequency, and spelling-to-sound consistency on the functional anatomy of reading. Neuron, 24(1), 205-218. https://doi.org/10.1016/S0896-6273(00)80833-8

Fonov, V., Evans, A. C., Botteron, K., Almli, C. R., McKinstry, R. C., \& Collins, D. L. (2011). Unbiased average age-appropriate atlases for pediatric studies. Neurolmage, 54(1), 313327. https://doi.org/10.1016/j.neuroimage.2010.07.033

Hagoort, P., Indefrey, P., Brown, C., Herzog, H., Steinmetz, H., \& Seitz, R. J. (1999). The neural circuitry involved in the reading of German words and pseudowords: A PET study. Journal of Cognitive Neuroscience, 11(4), 383-398. https://doi.org/10.1162/089892999563490

Hebb, A. O., \& Ojemann, G. A. (2013). The thalamus and language revisited. Brain and Language, 126(1), 99-108. https://doi.org/10.1016/j.bandl.2012.06.010

Heim, S., Alter, K., Ischebeck, A. K., Amunts, K., Eickhoff, S. B., Mohlberg, H., Zilles, K., von Cramon, D. Y., \& Friederici, A. D. (2005). The role of the left Brodmann's areas 44 and 45 in reading words and pseudowords. Brain Research. Cognitive Brain Research, 25(3), 982-993. https://doi.org/10.1016/j.cogbrainres.2005.09.022

Herbster, A. N., Mintun, M. A., Nebes, R. D., \& Becker, J. T. (1997). Regional cerebral blood flow during word and nonword reading. Human Brain Mapping, 5(2), 84-92. https://doi.org/10.1002/(sici)1097-0193(1997)5:2<84::aid-hbm2>3.0.co;2-i

Hickok, G. (2014). The architecture of speech production and the role of the phoneme in speech processing. Language, Cognition and Neuroscience, 29(1), 2-20.

https://doi.org/10.1080/01690965.2013.834370

Hintzen, A., Pelzer, E. A., \& Tittgemeyer, M. (2018). Thalamic interactions of cerebellum and basal ganglia. Brain Structure \& Function, 223(2), 569-587.

https://doi.org/10.1007/s00429-017-1584-y

Horn, A., \& Kühn, A. A. (2015). Lead-DBS: A toolbox for deep brain stimulation electrode localizations and visualizations. Neurolmage, 107, 127-135.

https://doi.org/10.1016/j.neuroimage.2014.12.002

Horn, A., Li, N., Dembek, T. A., Kappel, A., Boulay, C., Ewert, S., Tietze, A., Husch, A., Perera, 
T., Neumann, W.-J., Reisert, M., Si, H., Oostenveld, R., Rorden, C., Yeh, F.-C., Fang, Q., Herrington, T. M., Vorwerk, J., \& Kühn, A. A. (2019). Lead-DBS v2: Towards a comprehensive pipeline for deep brain stimulation imaging. Neurolmage, 184, 293-316. https://doi.org/10.1016/j.neuroimage.2018.08.068

Hwang, K., Bertolero, M. A., Liu, W. B., \& D’Esposito, M. (2017). The Human Thalamus Is an Integrative Hub for Functional Brain Networks. The Journal of Neuroscience, 37(23), 55945607. https://doi.org/10.1523/JNEUROSCI.0067-17.2017

Johnson, M. D., \& Ojemann, G. A. (2000). The role of the human thalamus in language and memory: Evidence from electrophysiological studies. Brain and Cognition, 42(2), 218-230. https://doi.org/10.1006/brcg.1999.1101

Juphard, A., Vidal, J. R., Perrone-Bertolotti, M., Minotti, L., Kahane, P., Lachaux, J.-P., \& Baciu,

Kühn, A. A., Williams, D., Kupsch, A., Limousin, P., Hariz, M., Schneider, G.-H., Yarrow, K., \& M. (2011). Direct Evidence for Two Different Neural Mechanisms for Reading Familiar and Brown, P. (2004). Event-related beta desynchronization in human subthalamic nucleus correlates with motor performance. Brain, 127(4), 735-746. https://doi.org/10.1093/brain/awh106

Levelt, W. J. M., Roelofs, A., \& Meyer, A. S. (1999). A theory of lexical access in speech production. In Behavioral and Brain Sciences (Vol. 22, Issue 1, pp. 1-75). https://doi.org/10.1017/S0140525X99001776

Lipski, W. J., Alhourani, A., Pirnia, T., Jones, P. W., Dastolfo-Hromack, C., Helou, L. B., Crammond, D. J., Shaiman, S., Dickey, M. W., Holt, L. L., Turner, R. S., Fiez, J. A., \& Richardson, R. M. (2018). Subthalamic nucleus neurons differentially encode early and late aspects of speech production. Journal of Neuroscience, 38(24), 5620-5631. https://doi.org/10.1523/JNEUROSCI.3480-17.2018

Llano, D. A. (2015). The Thalamus and Language. In G. Hickok \& S. L. Small (Eds.). Neurobiology of Language (pp. 95-114). Cambridge, MA: Academic Press.

Macchi, G., \& Jones, E. G. (1997). Toward an agreement on terminology of nuclear and subnuclear divisions of the motor thalamus. Journal of Neurosurgery, 86(1), 77-92. 
Mechelli, A., Gorno-Tempini, M. L., \& Price, C. J. (2003). Neuroimaging studies of word and pseudoword reading: Consistencies, inconsistencies, and limitations. Journal of Cognitive Neuroscience, 15(2), 260-271. https://doi.org/10.1162/089892903321208196

Moore, M. W., Fiez, J. A., \& Tompkins, C. A. (2017). Consonant Age-of-Acquisition Effects in Nonword Repetition Are Not Articulatory in Nature. Journal of Speech, Language, and Hearing Research, 60(11), 3198-3212. https://doi.org/10.1044/2017_JSLHR-L-16-0359

Paradiso, G. (2004). Involvement of human thalamus in the preparation of self-paced

Ray, S., Crone, N. E., Niebur, E., Franaszczuk, P. J., \& Hsiao, S. S. (2008). Neural correlates of high-gamma oscillations $(60-200 \mathrm{~Hz})$ in macaque local field potentials and their potential implications in electrocorticography. The Journal of Neuroscience : The Official Journal of the Society for Neuroscience, 28(45), 11526-11536. https://doi.org/10.1523/JNEUROSCI.2848-08.2008

Ray, S., \& Maunsell, J. H. R. (2011). Different Origins of Gamma Rhythm and High-Gamma Activity in Macaque Visual Cortex. PLoS Biology, 9(4), e1000610. https://doi.org/10.1371/journal.pbio.1000610

Tiedt, H. O., Ehlen, F., Krugel, L. K., Horn, A., Kühn, A. A., \& Klostermann, F. (2017). Subcortical roles in lexical task processing: Inferences from thalamic and subthalamic event-related potentials. Human Brain Mapping, 38(1), 370-383. https://doi.org/10.1002/hbm.23366

Tzagarakis, C., Ince, N. F., Leuthold, A. C., \& Pellizzer, G. (2010). Beta-band activity during motor planning reflects response uncertainty. The Journal of Neuroscience: The Official

748 Wahl, M., Marzinzik, F., Friederici, A. D., Hahne, A., Kupsch, A., Schneider, G. H., Saddy, D., 
Curio, G., \& Klostermann, F. (2008). The Human Thalamus Processes Syntactic and

750 Semantic Language Violations. Neuron, 59(5), 695-707.

751 https://doi.org/10.1016/j.neuron.2008.07.011

752 Xu, B. (2001). Conjoint and Extended Neural Networks for the Computation of Speech Codes:

753 The Neural Basis of Selective Impairment in Reading Words and Pseudowords. Cerebral

754 Cortex, 11(3), 267-277. https://doi.org/10.1093/cercor/11.3.267

755 Zhang, D., Snyder, A. Z., Shimony, J. S., Fox, M. D., \& Raichle, M. E. (2010). Noninvasive

756 Functional and Structural Connectivity Mapping of the Human Thalamocortical System.

757 Cerebral Cortex, 20(5), 1187-1194. https://doi.org/10.1093/cercor/bhp182 
a

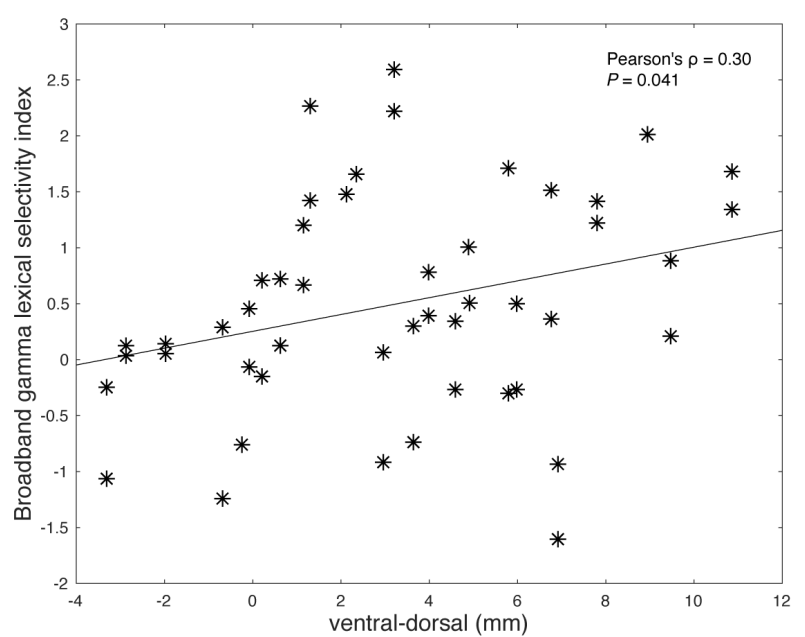

b

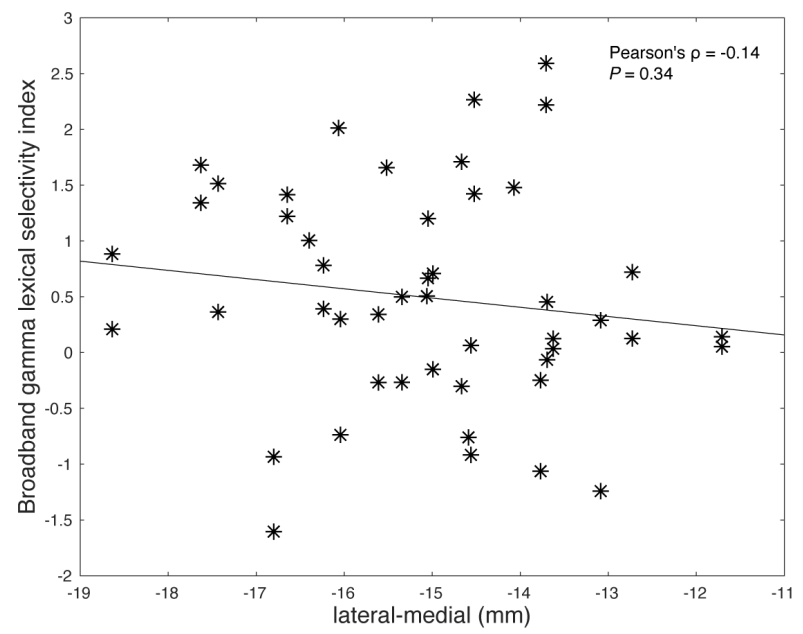

Supplementary Figure 1: Correlation between left thalamic broadband gamma lexical selectivity and recording locations along ventral-dorsal and lateral-medial axes.

769 Broadband gamma lexical selectivity indexes of left side lead recordings that showed significant 770 task-related broadband gamma increase responses are correlated (Pearson's correlation, $771 \alpha=0.05)$ with ventral-dorsal locations (a) and lateral-medial locations (b) of the recordings, 772 respectively. 
a

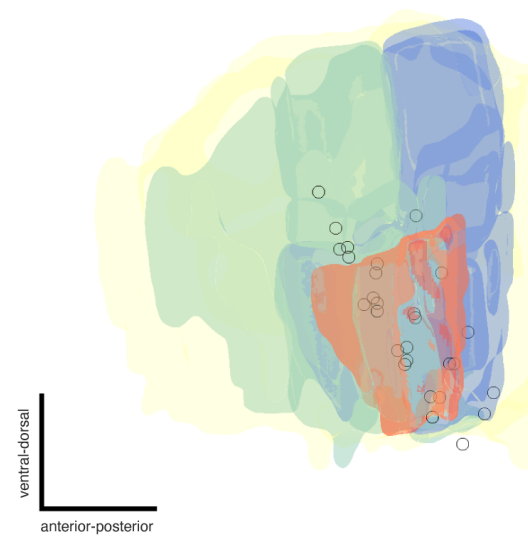

773

774

775

776

777

778

779

780

781

782

783

784

785

786 VLp are blue-colored. b



Supplementary Figure 2: Broadband gamma lexical selectivity does not depend on anterior-posterior location of the recording in the right thalamus. a Right side lead recording sites are flipped to the left and plotted together with anatomical structures (thalamus in yellow, VA and VLa in green, VLp in blue, and Vim in orange), viewed from a lateral direction. Recording sites where significantly nonword-selective recordings in terms of broadband gamma response amplitudes (broadband gamma lexical selectivity index $>1.645$ based on normal approximation of $\mathrm{t}$-distribution) were observed are filled with black $(\mathrm{n}=0)$, and remaining recording sites are shown in open circles. b Broadband gamma lexical selectivity indexes of right side lead recordings that showed significant task-related broadband gamma increase responses are correlated (Pearson's correlation, $\alpha=0.05$ ) with recording locations along the anterior-posterior axis. Recordings inside VA or VLa are green-colored and recordings inside 
a

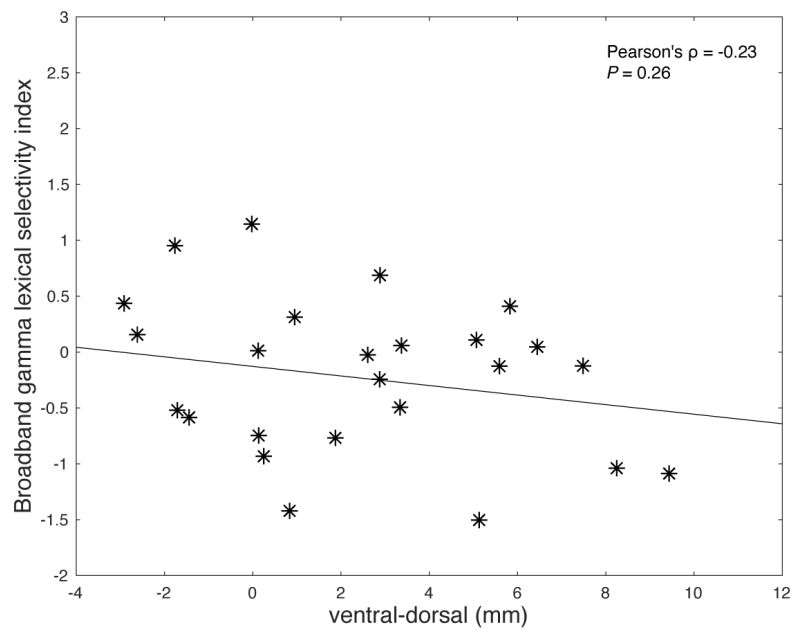

b

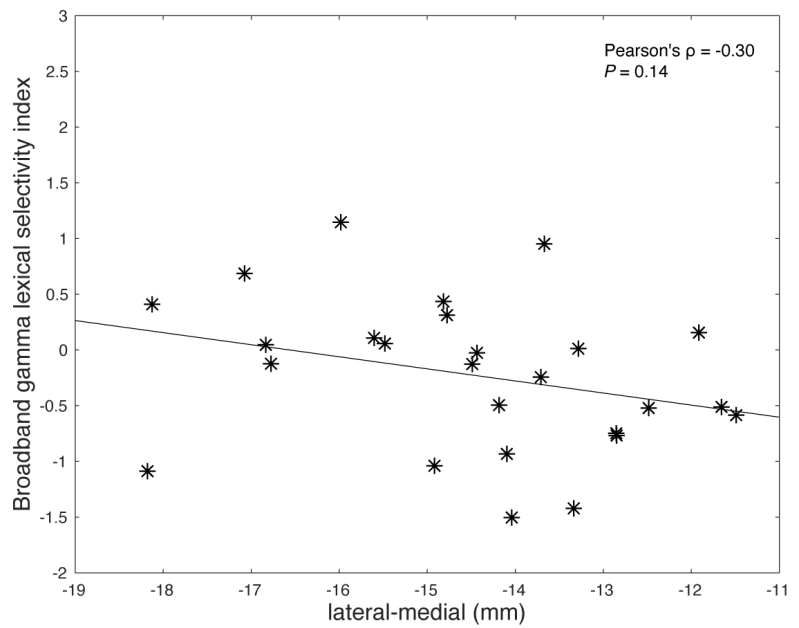

Supplementary Figure 3: Correlation between right thalamic broadband gamma lexical selectivity and recording locations along ventral-dorsal and lateral-medial axes. Broadband gamma lexical selectivity indexes of right side lead recordings that showed significant task-related broadband gamma increase responses are correlated (Pearson's correlation, $\alpha=0.05)$ with ventral-dorsal locations (a) and lateral-medial locations (b) of the recordings, respectively. 
a

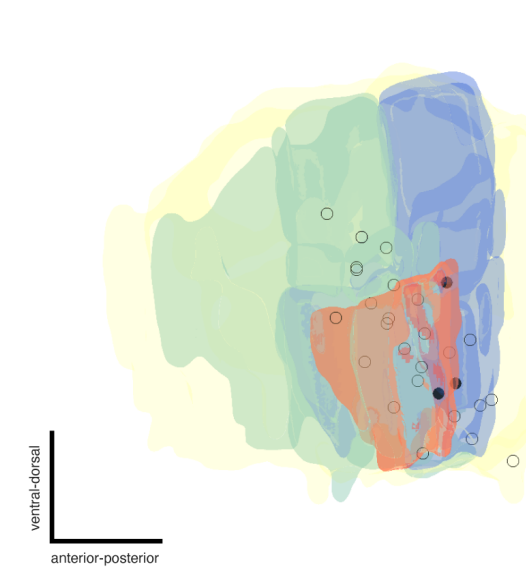

C

795

796

797

798

799

800

801

802

803

804

805

806

807

808

809
Left

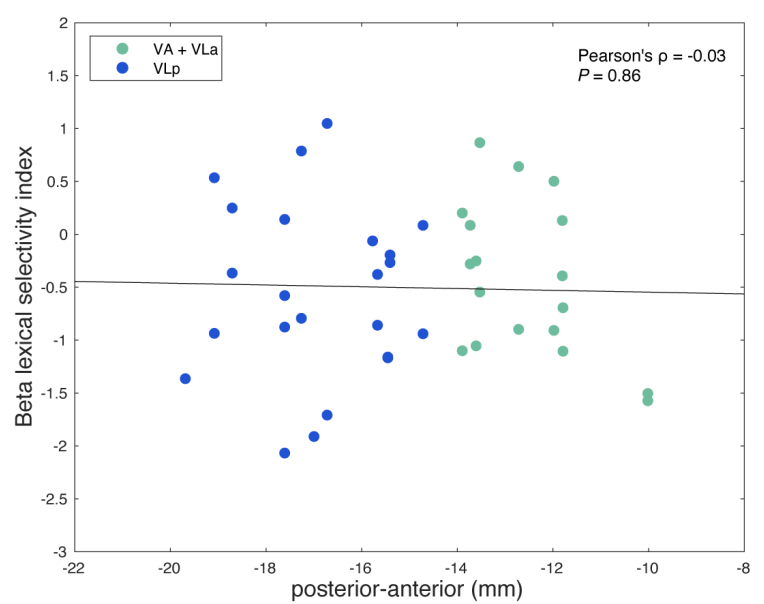

b

Right
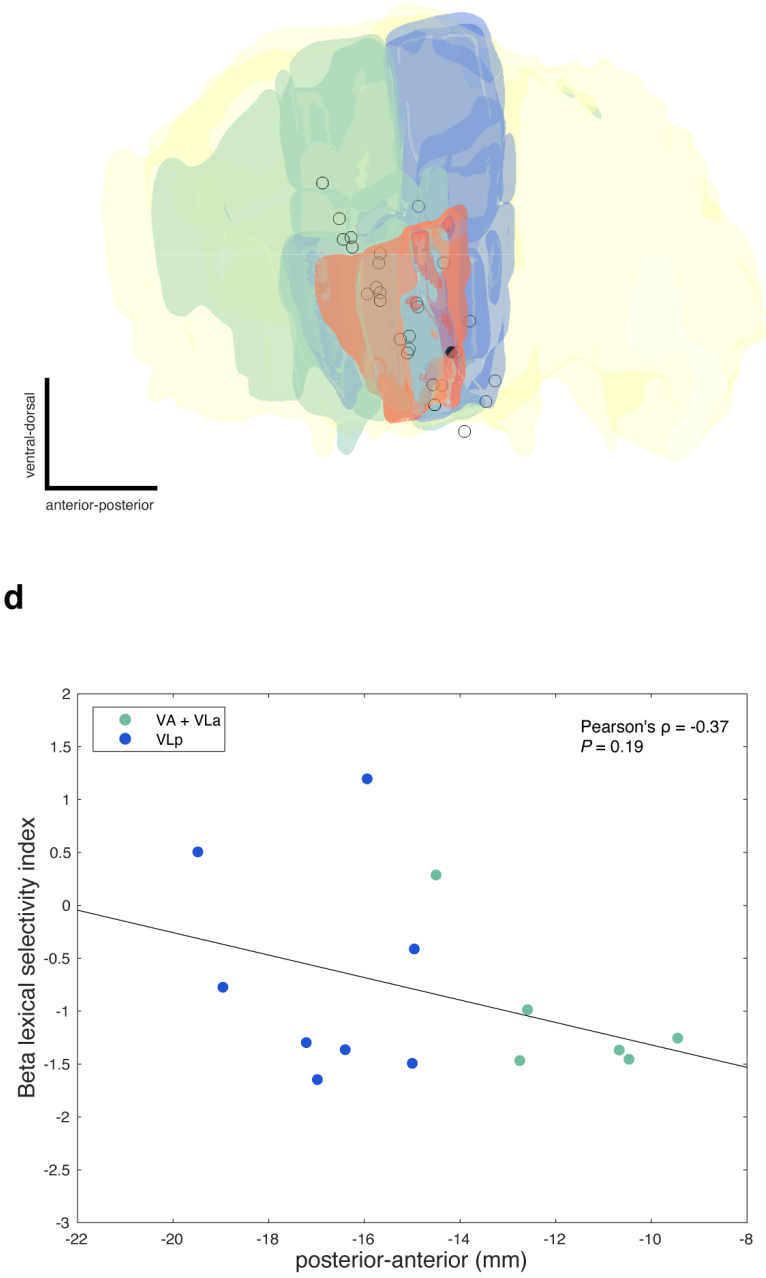

Supplementary Figure 4: Beta lexical selectivity does not depend on anterior-posterior location of the recording in either side. a Left side lead recording sites are plotted together with anatomical structures (thalamus in yellow, VA and VLa in green, VLp in blue, and Vim in orange), viewed from a lateral direction. Recording sites where significantly nonword-selective recordings in terms of beta response amplitudes (beta lexical selectivity index<-1.645 based on normal approximation of t-distribution) were observed in either session are filled with black, and remaining recording sites are shown in open circles. b Right side lead recording sites are flipped to the left and plotted in the same way. c Beta lexical selectivity indexes of left side lead recordings that showed significant task-related beta decrease responses are correlated (Pearson's correlation, $\alpha=0.05$ ) with recording locations along the anterior-posterior axis. $\mathbf{d}$ Beta lexical selectivity indexes of right side lead recordings that showed significant task-related beta decrease responses are correlated (Pearson's correlation, $\alpha=0.05$ ) with recording locations along the anterior-posterior axis. In $\mathbf{c}$ and $\mathbf{d}$, recordings inside VA or VLa are green-colored and recordings inside VLp are blue-colored. 
a

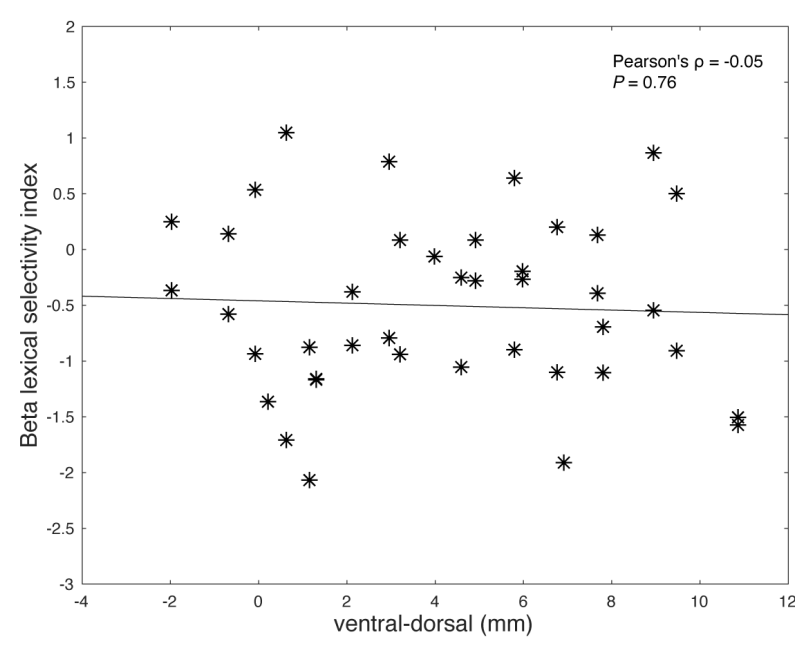

C

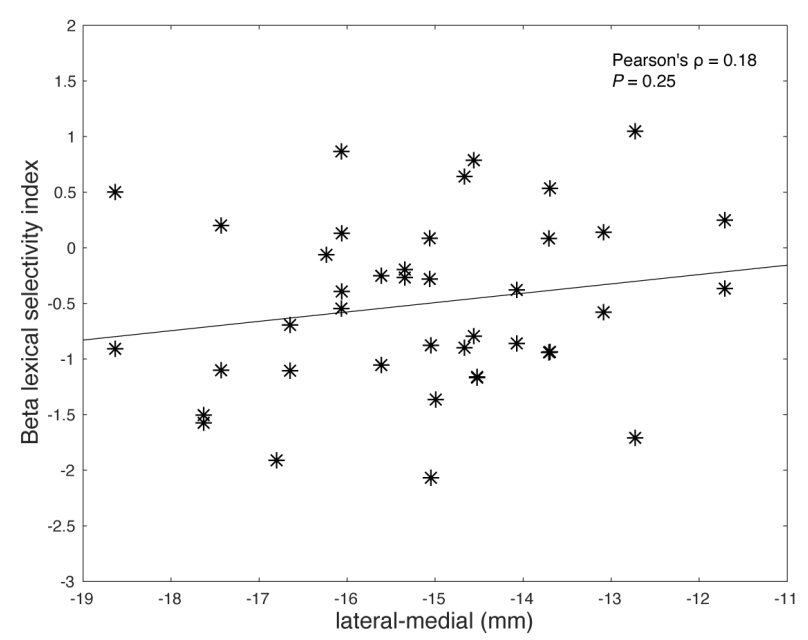

b

Right

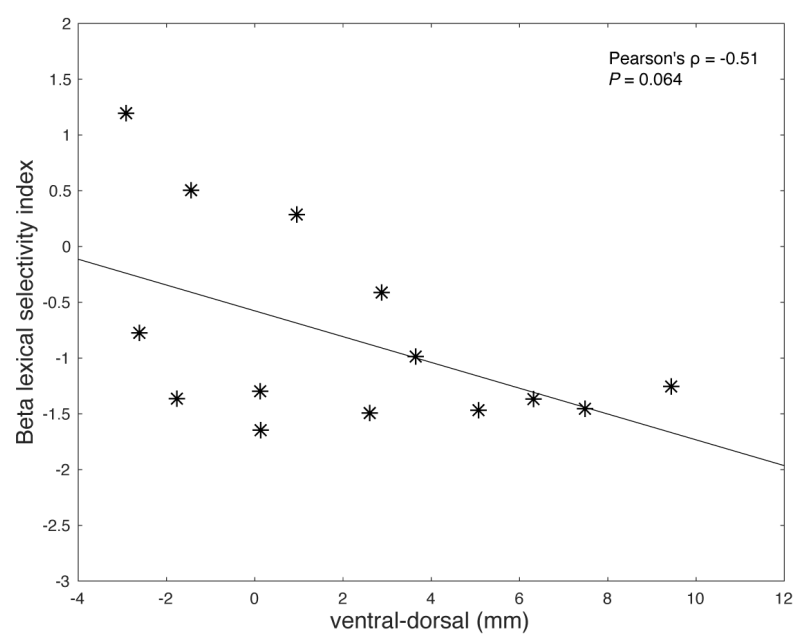

d

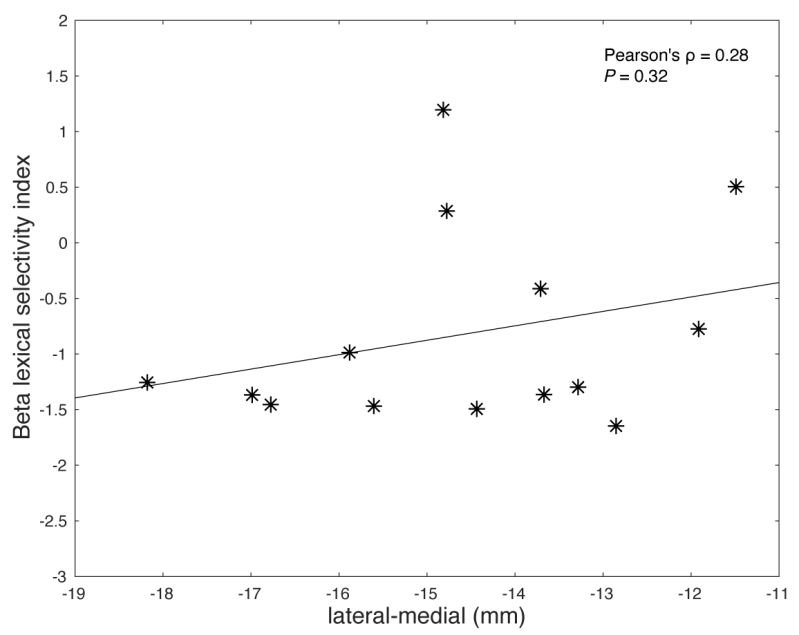

Supplementary Figure 5: Correlation between thalamic beta lexical selectivity and recording locations along ventral-dorsal and lateral-medial axes. a, c Beta lexical selectivity indexes of left side lead recordings that showed significant task-related beta decrease responses are correlated (Pearson's correlation, $\alpha=0.05)$ with ventral-dorsal locations $(a)$ and lateral-medial locations (c) of the recordings, respectively. b, d Beta lexical selectivity indexes of right side lead recordings that showed significant task-related beta decrease responses are correlated (Pearson's correlation, $\alpha=0.05$ ) with ventral-dorsal locations (b) and lateral-medial locations (d) of the recordings, respectively. 
Supplementary Table 1: Broadband gamma increase response and beta decrease

820 response differ in locking properties.

821

\begin{tabular}{|l|l|l|l|l|}
\hline Response type & $\begin{array}{l}\text { Number of } \\
\text { recordings }\end{array}$ & $\begin{array}{l}\text { Stimulus- } \\
\text { locked }(\%)\end{array}$ & $\begin{array}{l}\text { Speech- } \\
\text { locked (\%) }\end{array}$ & $\begin{array}{l}\text { Not locked } \\
(\%)\end{array}$ \\
\hline Broadband gamma increase response & 91 & $19(21)$ & $70(77)$ & $2(2)$ \\
\hline Beta decrease response & 66 & $43(65)$ & $19(29)$ & $4(6)$ \\
\hline$X^{2}$ & - & 31.4 & 36.1 & 1.55 \\
\hline$P$ & - & $<10^{-5}$ & $<10^{-5}$ & 0.21 \\
\hline
\end{tabular}

822 Supplementary Table 2: Locking properties of broadband gamma increase response and

823 beta decrease response do not differ between recording sides.

\begin{tabular}{|c|c|c|c|c|}
\hline Response type & $\begin{array}{l}\text { Number of } \\
\text { recordings }\end{array}$ & $\begin{array}{l}\text { Stimulus- } \\
\text { locked (\%) }\end{array}$ & $\begin{array}{l}\text { Speech- } \\
\text { locked (\%) }\end{array}$ & $\begin{array}{l}\text { Not locked } \\
(\%)\end{array}$ \\
\hline \multicolumn{5}{|c|}{ Broadband gamma increase response } \\
\hline Left & 59 & $10(17)$ & $47(80)$ & $2(3)$ \\
\hline Right & 32 & $9(28)$ & $23(72)$ & $0(0)$ \\
\hline$x^{2}$ & \multicolumn{4}{|l|}{2.49} \\
\hline$P$ & \multicolumn{4}{|l|}{0.29} \\
\hline \multicolumn{5}{|c|}{ Beta decrease response } \\
\hline Left & 49 & $31(63)$ & $14(29)$ & $4(8)$ \\
\hline Right & 17 & $12(71)$ & $5(29)$ & $0(0)$ \\
\hline$x^{2}$ & \multicolumn{4}{|l|}{1.50} \\
\hline$P$ & \multicolumn{4}{|l|}{0.47} \\
\hline
\end{tabular}

824 
825 Supplementary Table 3: Stimulus lists.

\begin{tabular}{|c|c|c|c|c|c|c|c|}
\hline \multicolumn{2}{|c|}{ Stimulus list 1} & \multicolumn{2}{|c|}{ Stimulus list 2} & \multicolumn{2}{|c|}{ Stimulus list 3} & \multicolumn{2}{|c|}{ Stimulus list 4} \\
\hline First 60 & Last 60 & First 60 & Last 60 & First 60 & Last 60 & First 60 & Last 60 \\
\hline daif & daif & dight & dight & deave & deave & dipe & dipe \\
\hline van & dutt & void & dute & vowed & doif & vote & doan \\
\hline dutt & foat & dute & fape & doif & feam & doan & fode \\
\hline vied & fute & vet & foon & vat & feeve & vain & fett \\
\hline foat & meef & fape & mide & feam & vune & fode & meave \\
\hline town & mipe & type & tep & tied & mafe & towed & mep \\
\hline fute & neave & foon & veem & feeve & mupe & fett & neam \\
\hline teed & nime & tune & naid & team & nipe & time & noit \\
\hline meef & paim & mide & noop & vune & noove & meave & peff \\
\hline putt & pote & pain & pime & poof & pode & pine & poom \\
\hline mipe & teeve & tep & peaf & mafe & poun & mep & taid \\
\hline pout & toff & peeve & taff & pave & teep & phone & tife \\
\hline neave & vapp & veem & toove & mupe & tem & neam & vadd \\
\hline note & vife & node & vade & need & vight & paid & vate \\
\hline nime & vean & naid & vome & nipe & ved & noit & veet \\
\hline moon & laz & mood & zool & moat & soith & neat & leeth \\
\hline paim & saith & noop & loath & noove & luth & peff & sish \\
\hline mauve & lesh & mean & luss & main & losh & mode & luthe \\
\hline pote & rel & pime & rall & pode & rith & poom & ral \\
\hline knife & reez & knot & suzz & knight & ruzz & might & riz \\
\hline teeve & shuss & peaf & saze & poun & sar & taid & soush \\
\hline fought & shule & fight & seash & foot & shoss & knit & shar \\
\hline toff & sosh & taff & shez & teep & shiz & tife & shoth \\
\hline five & sithe & feed & shile & foam & sile & food & thaze \\
\hline vapp & thace & toove & thar & tem & thale & vadd & theal \\
\hline fade & thauz & fame & thil & fain & thall & fate & sule \\
\hline vife & thole & vade & thure & vight & thice & vate & sazz \\
\hline deep & thoss & dine & thush & doubt & thel & dive & thosh \\
\hline vean & zal & vome & zel & ved & zoice & veet & zoil \\
\hline dawn & zole & date & soth & dime & zus & dean & zil \\
\hline laz & daif & zool & dight & soith & deave & leeth & dipe \\
\hline
\end{tabular}




\begin{tabular}{|c|c|c|c|c|c|c|c|}
\hline zoom & dutt & zone & dute & zip & doif & zap & doan \\
\hline saith & foat & loath & fape & luth & feam & sish & fode \\
\hline thought & fute & thud & foon & thumb & feeve & thief & fett \\
\hline lesh & meef & luss & mide & losh & vune & luthe & meave \\
\hline thin & mipe & theme & tep & that & mafe & them & mep \\
\hline rel & neave & rall & veem & rith & mupe & ral & neam \\
\hline this & nime & wrath & naid & zeal & nipe & thus & noit \\
\hline reez & paim & suzz & noop & ruzz & noove & riz & peff \\
\hline shoes & pote & these & pime & those & pode & thaws & poom \\
\hline shuss & teeve & saze & peaf & sar & poun & soush & taid \\
\hline shawl & toff & sure & taff & south & teep & shies & tife \\
\hline shule & vapp & seash & toove & shoss & tem & shar & vadd \\
\hline sell & vife & soar & vade & sill & vight & sews & vate \\
\hline sosh & vean & shez & vome & shiz & ved & shoth & veet \\
\hline sear & laz & size & zool & shore & soith & sale & leeth \\
\hline sithe & saith & shile & loath & sile & luth & thaze & sish \\
\hline sash & lesh & shell & luss & shale & losh & share & luthe \\
\hline thace & rel & thar & rall & thale & rith & theal & ral \\
\hline rush & reez & seal & suzz & role & ruzz & lace & riz \\
\hline thauz & shuss & thil & saze & thall & sar & sule & soush \\
\hline rise & shule & rose & seash & rash & shoss & lash & shar \\
\hline thole & sosh & thure & shez & thice & shiz & sazz & shoth \\
\hline race & sithe & rail & shile & raise & sile & lore & thaze \\
\hline thoss & thace & thush & thar & thel & thale & thosh & theal \\
\hline loathe & thauz & loss & thil & lure & thall & lose & sule \\
\hline zal & thole & zel & thure & zoice & thice & zoil & sazz \\
\hline laze & thoss & lies & thush & lows & thel & rule & thosh \\
\hline zole & zal & soth & zel & zus & zoice & zil & zoil \\
\hline laws & zole & leash & soth & lice & zus & rice & zil \\
\hline
\end{tabular}


828 Supplementary Table 4: $\boldsymbol{P}$ values of statistical tests comparing psycholinguistic

829 parameters between word and nonword stimuli.

\begin{tabular}{|l|c|c|c|c|}
\hline & Stimulus list 1 & Stimulus list 2 & Stimulus list 3 & Stimulus list 4 \\
\hline $\begin{array}{l}\text { Weighted phonological } \\
\text { neighborhood density }\end{array}$ & 0.23 & 0.43 & 0.88 & 0.86 \\
\hline Summed bigram frequency & 0.97 & 0.51 & 0.39 & 0.32 \\
\hline Summed phoneme probability & 0.47 & 0.33 & 0.37 & 0.51 \\
\hline Summed biphone probability & 0.65 & 0.65 & 0.65 & 0.90 \\
\hline
\end{tabular}

830 Two-tailed two-sample t-test, $n=60, \alpha=0.05$. 